\title{
Filtered Dynamic Inversion for Altitude Control of Fixed-Wing Unmanned Air Vehicles
}

\author{
Jon Mullen, Sean C. C. Bailey, and Jesse B. Hoagg* \\ Department of Mechanical Engineering, University of Kentucky, Lexington, KY 40506-0503
}

\begin{abstract}
Instrumented unmanned air vehicles (UAVs) represent a new way of conducting atmospheric science, particularly within the atmospheric boundary layer where the air is turbulent. However, using autonomous UAVs for airborne measurement requires active control methods capable of following altitude commands despite unknown and turbulent disturbances to the air. Filtered dynamic inversion (FDI) is a control method with desirable command-following and disturbance-rejection properties for this application. FDI requires limited model information and is thus robust to parametric uncertainty, which arises in modeling UAV dynamics. In this paper, FDI is implemented in an altitude-flight-control system for an autonomous fixed-wing UAV. The control system is validated in simulation with a nonlinear dynamic model of a small fixed-wing UAV. The control system is also implemented and validated in flight experiments with turbulent wind conditions. Experimental results show that FDI yields improved altitude and pitch command following as compared to a classical (e.g., proportional-integral) flight-control system. In particular, experimental data demonstrate that the average power of the altitude and pitch command-following errors with FDI is smaller than those with proportionalintegral control.
\end{abstract}

Keywords: UAV; dynamic inversion; flight control

\section{Introduction}

Advances in sensor miniaturization have improved the viability of small unmanned air vehicles (UAVs) for a wide range of applications, including precision agriculture, search and rescue, and aerial surveillance. In addition to these vision-sensing applications, small UAVs can also be used to take meteorological measurements $[1,2,3,4,5]$.

For several decades, manned aircraft have been used for atmospheric research such as conducting weather reconnaissance; measuring wind, temperature, and humidity profiles $[6,7,8]$; measuring atmospheric turbulence $[9]$; and tracking pollutant concentrations [10]. Small UAVs have advantages over manned aircraft, including reduced operational costs and the ability to operate and obtain measurements close to the Earth's surface [11]. Despite their potential, the use of UAVs for atmospheric research is still in its infancy, focusing on remotely piloted UAVs for obtaining wind, temperature, and humidity profiles $[12,13]$. Measurements during autonomous flight have been reported in Refs. [1, 4, 14, 15, 16].

Two of the traits, namely, small size and light weight, that are driving the increase in UAV usage for meteorological measurements also introduce challenges. Specifically, small lightweight UAVs are susceptible to the quasirandom forcing introduced by turbulence in the atmospheric boundary layer near the Earth's surface [17]. This boundary layer is the predominant region of interest for studying transport processes between the surface and the atmosphere. However, turbulence in the boundary layer

*Corresponding author; email: jhoagg@engr.uky.edu can disturb a UAV's flight path and, thus, adversely impact the statistical accuracy of measurements from onboard sensors.

To improve the suitability of small lightweight UAVs as sensor platforms, it is necessary to improve their ability to correct for the impact of wind gusts induced by the turbulence. One approach is to improve the capabilities of the flight-control system. This paper examines the use of filtered dynamic inversion (FDI) for altitude control of a small fixed-wing UAV. FDI is a control method for highly uncertain minimum-phase linear dynamic systems, and is effective for command following in the presence of unmeasured disturbances [18, 19]. In particular, Ref. [18] shows that for sufficiently large choice of a single control parameter, FDI makes the average power of the command-following error arbitrarily small despite unmeasured disturbances (e.g., turbulent wind). FDI is also effective for systems with nonlinear dynamics [20]. In this paper, an FDI control system is designed and implemented on a small fixed-wing UAV to achieve effective altitude command following in the presence of turbulent wind.

The main contributions of this paper include the design of an FDI flight-control system for a small fixed-wing UAV, and the testing and validation of this FDI control system through numerical simulations and flight experiments. The flight experiments and numerical simulations demonstrate that FDI reduces the altitude command-following error as compared to a classical (e.g., proportional-integral) controller. Experimental data demonstrate that the average power of the altitude error with FDI is smaller than those with proportional-integral control. 


\section{Nomenclature}

\section{Filtered Dynamic Inversion}

$\begin{array}{ll}A, B, C & \text { state-variable system matrices } \\ x & \text { state variable } \\ u & \text { control input } \\ w & \text { disturbance input } \\ y & \text { output } \\ H_{d} & \text { first nonzero Markov parameter } \\ d & \text { relative degree } \\ \mathbf{p} \triangleq \mathrm{d} / \mathrm{d} t & \text { differential operator } \\ \alpha_{\mathrm{m}}, \beta_{\mathrm{m}} & \text { reference-model polynomial matrices } \\ r & \text { reference-model input } \\ y_{\mathrm{m}} & \text { reference-model output } \\ z \triangleq y-y \mathrm{~m} & \text { error } \\ \mathcal{P}_{z} & \text { average power of } z \\ u_{*} & \text { ideal dynamic inversion control } \\ \rho & \text { FDI controller order } \\ k & \text { FDI parameter } \\ \eta_{k} & \text { FDI polynomial }\end{array}$

\section{Nonlinear UAV Dynamics}

$F_{\text {I }}$

$o_{\mathrm{I}}$

$\hat{\imath}_{\mathrm{I}}, \hat{\jmath}_{\mathrm{I}}, \hat{k}_{\mathrm{I}}$

$F_{\mathrm{B}}$

$o_{\mathrm{B}}$

$\hat{\imath}_{\mathrm{B}}, \hat{\jmath}_{\mathrm{B}}, \hat{k}_{\mathrm{B}}$

$\vec{r}$

$\underset{\rightarrow}{X}, Y, Z$

$\vec{v}$

$v_{\mathrm{B}}$

$U, V, W$

$\vec{\omega}$

$\omega_{\mathrm{B}}$

$P, Q, R$

$\phi, \theta, \psi$

$m$

$\vec{I}$

$I_{\mathrm{xx}}, I_{\mathrm{yy}}, I_{\mathrm{zz}}$

$I_{\mathrm{xz}}$

$\overrightarrow{F_{\mathrm{a}}}$

$X_{\mathrm{a}}, Y_{\mathrm{a}}, Z_{\mathrm{a}}$

$\overrightarrow{F_{\mathrm{T}}}$

$X_{\mathrm{T}}, Y_{\mathrm{T}}, Z_{\mathrm{T}}$

$\vec{g}$

$\vec{M}_{\mathrm{c}}$

$L, M, N$

$\overrightarrow{v_{\mathrm{w}}}$

$\overrightarrow{v_{\mathrm{r}}} \triangleq \vec{v}-\overrightarrow{v_{\mathrm{w}}}$

$U_{\mathrm{r}}, V_{\mathrm{r}}, W_{\mathrm{r}}$

$\alpha$

\section{inertial frame}

center of $F_{\mathrm{I}}$

orthogonal unit vectors of $F_{\mathrm{I}}$

body frame

center of $F_{\mathrm{B}}$, which is the center of mass

orthogonal unit vectors of $F_{\mathrm{B}}$

position of $o_{\mathrm{B}}$ relative to $o_{\mathrm{I}}(\mathrm{m})$

components of $\vec{r}$ resolved in $F_{\mathrm{I}}(\mathrm{m})$

velocity of $o_{\mathrm{B}}$ relative to $o_{\mathrm{I}}$ with respect

to $F_{\mathrm{I}}(\mathrm{m} / \mathrm{s})$

$\vec{v}$ resolved in $F_{\mathrm{B}}(\mathrm{m} / \mathrm{s})$

components of $v_{\mathrm{B}}(\mathrm{m} / \mathrm{s})$

angular velocity of $F_{\mathrm{B}}$ relative to $F_{\mathrm{I}}(\mathrm{rad} / \mathrm{s})$

$\vec{\omega}$ resolved in $F_{\mathrm{B}}(\mathrm{rad} / \mathrm{s})$

components of $\omega_{\mathrm{B}}(\mathrm{rad} / \mathrm{s})$

yaw, pitch, roll Euler angles (rad)

mass (kg)

physical inertia matrix $\left(\mathrm{kg} \cdot \mathrm{m}^{2}\right)$

moments of inertia $\left(\mathrm{kg} \cdot \mathrm{m}^{2}\right)$

product of inertia $\left(\mathrm{kg} \cdot \mathrm{m}^{2}\right)$

aerodynamic force $(\mathrm{N})$

components of $\vec{F}_{\mathrm{a}}$ resolved in $F_{\mathrm{B}}(\mathrm{N})$

thrust force $(\mathrm{N})$

components of $\overrightarrow{F_{\mathrm{T}}}$ resolved in $F_{\mathrm{B}}(\mathrm{N})$

acceleration due to gravity $\left(\mathrm{m} / \mathrm{s}^{2}\right)$

moment due to aerodynamic force $(\mathrm{N} \cdot \mathrm{m})$

components of $\vec{M}_{\mathrm{c}}$ resolved in $F_{\mathrm{B}}(\mathrm{N} \cdot \mathrm{m})$

wind velocity $(\mathrm{m} / \mathrm{s})$

relative velocity $(\mathrm{m} / \mathrm{s})$

components of $\overrightarrow{v_{\mathrm{r}}}$ resolved in $F_{\mathrm{B}}(\mathrm{m} / \mathrm{s})$

angle of attack (rad) $\beta \quad$ sideslip angle (rad)

$V_{\mathrm{T}} \quad$ airspeed $(\mathrm{m} / \mathrm{s})$

$\delta_{\mathrm{e}} \quad$ elevator deflection angle (rad)

$\delta_{\mathrm{r}} \quad$ rudder deflection angle ( $\mathrm{rad}$ )

$\delta_{\mathrm{a}} \quad$ aileron deflection angle (rad)

FDI Flight Control System

$h \triangleq-Z \quad$ altitude $(\mathrm{m})$

$h_{\mathrm{d}} \quad$ altitude command $(\mathrm{m})$

$U_{\mathrm{d}} \quad$ speed command $(\mathrm{m} / \mathrm{s})$

$\theta_{\mathrm{d}} \quad$ pitch command (rad)

$u_{\mathrm{T}} \quad$ throttle command

$u_{\mathrm{e}} \quad$ elevator command (rad)

$\tilde{\theta} \triangleq \theta_{\mathrm{d}}-\theta \quad$ pitch error $(\mathrm{rad})$

$\mathcal{P}_{h}\left(t_{1}, t_{0}\right) \quad$ average power of the altitude error on the $\left[t_{0}, t_{1}\right)$ time interval $\left(\mathrm{m}^{2}\right)$

$\mathcal{P}_{\theta}\left(t_{1}, t_{0}\right) \quad$ average power of the pitch error on the $\left[t_{0}, t_{1}\right)$ time interval $\left(\mathrm{rad}^{2}\right)$

$k_{h, \mathrm{p}} \quad$ altitude controller proportional gain

$k_{h, \mathrm{i}} \quad$ altitude controller integral gain

$g_{\mathrm{T}} \quad$ engine gain

$k_{\mathrm{T}, \mathrm{p}} \quad$ speed controller proportional gain

$k_{\mathrm{T}, \mathrm{i}} \quad$ speed controller integral gain

$C_{k} \quad$ transfer function for FDI pitch controller

Linearized Equations of Motion for Longitudinal Flight

$U_{0} \quad$ equilibrium $\hat{\imath}_{\mathrm{B}}$-direction velocity $(\mathrm{m} / \mathrm{s})$

$W_{0} \quad$ equilibrium $\hat{k}_{\mathrm{B}}$-direction velocity $(\mathrm{m} / \mathrm{s})$

$\theta_{0} \quad$ equilibrium pitch (rad)

$X_{\mathrm{T}, 0} \quad$ equilibrium $\hat{\imath}_{\mathrm{B}}$-direction thrust $(\mathrm{N})$

$\delta_{\mathrm{e}, 0} \quad$ equilibrium elevator deflection ( $\mathrm{rad}$ )

$\Delta U \quad \hat{\imath}_{\mathrm{B}}$-direction velocity perturbation $(\mathrm{m} / \mathrm{s})$

$\Delta W \quad \hat{k}_{\mathrm{B}}$-direction velocity perturbation $(\mathrm{m} / \mathrm{s})$

$\Delta \theta \quad$ pitch perturbation ( $\mathrm{rad}$ )

$\Delta X_{\mathrm{T}} \quad \hat{\imath}_{\mathrm{B}}$-direction thrust perturbation $(\mathrm{N})$

$\Delta \delta_{\mathrm{e}} \quad$ elevator deflection perturbation (rad)

$x_{1} \quad$ state variable for longitudinal flight

$A_{1} \quad$ dynamics matrix for longitudinal flight

$B_{1, X_{\mathrm{T}}} \quad \Delta X_{\mathrm{T}}$ input matrix for longitudinal flight

$B_{1, \delta_{\mathrm{e}}} \quad \Delta \delta_{\mathrm{e}}$ input matrix for longitudinal flight

$\tau_{\mathrm{e}} \quad$ elevator servomechanism time constant (s)

$\Delta U_{\mathrm{d}} \quad$ speed command perturbation $(\mathrm{m} / \mathrm{s})$

$\Delta u_{\mathrm{e}} \quad$ elevator command perturbation (rad)

$G \quad$ transfer function for linearized longitudinal

$\tilde{G}_{k} \quad$ closed-loop transfer function for linearized dynamics from $\Delta u_{\mathrm{e}}$ to $\tilde{\theta}$ with FDI control

\section{Other Parameters}

$b_{\mathrm{r}} \quad$ wing $\operatorname{span}(\mathrm{m})$

$c_{\mathrm{r}} \quad$ mean cord length $(\mathrm{m})$

$S_{\mathrm{r}} \quad$ planform area $\left(\mathrm{m}^{2}\right)$

$\rho_{\mathrm{a}} \quad$ density of air $\left(\mathrm{kg} / \mathrm{m}^{2}\right)$

$k_{\theta, \mathrm{p}} \quad$ baseline pitch controller proportional gain

$k_{\theta, \mathrm{i}} \quad$ baseline pitch controller integral gain

$k_{\phi, \mathrm{p}} \quad$ roll controller proportional gain

$k_{\phi, \mathrm{i}} \quad$ roll controller integral gain

$T_{\mathrm{s}} \quad$ sample time $(\mathrm{s})$ 


\section{Filtered Dynamic Inversion}

FDI is a feedback control method for uncertain linear time-invariant dynamic systems that are minimum phase (i.e., invariant zeros contained in the open-left-half complex plane) and potentially subject to unmeasured disturbances (e.g., turbulent wind). To illustrate FDI, consider the state-variable dynamic system

$$
\begin{aligned}
\dot{x}(t) & =A x(t)+B u(t)+w(t), \\
y(t) & =C x(t),
\end{aligned}
$$

where $t \geq 0, A \in \mathbb{R}^{n \times n}, B \in \mathbb{R}^{n \times m}, C \in \mathbb{R}^{m \times n}, x(0) \in \mathbb{R}^{n}$ is the initial condition, $x(t) \in \mathbb{R}^{n}$ is the state, $u(t) \in$ $\mathbb{R}^{m}$ is the control, $y(t) \in \mathbb{R}^{m}$ is the output, $w(t) \in \mathbb{R}^{n}$ is an unmeasured disturbance, and $(A, B, C)$ is controllable and observable. We assume that the invariant zeros of $(A, B, C)$ are contained in the open-left-half complex plane. The relative degree $d$ from $u$ to $y$ is the smallest integer $i$ such that the $i$ th Markov parameter $H_{i} \triangleq C A^{i-1} B$ is nonzero. FDI relies on knowledge of $d$ and $H_{d}$; however, $A, B$, and $C$ are otherwise unknown.

Let $\alpha_{\mathrm{m}}$ be a real monic $m \times m$ polynomial matrix with degree $d$, where $\operatorname{det} \alpha_{\mathrm{m}}$ is Hurwitz (i.e., the roots are in the open-left-half complex plane), and let $\beta_{\mathrm{m}}$ be a real $m \times m$ polynomial matrix with degree not greater than $d$. Next, let $\mathbf{p} \triangleq \mathrm{d} / \mathrm{d} t$ denote the differential operator, and consider the reference-model dynamics $\alpha_{\mathrm{m}}(\mathbf{p}) y_{\mathrm{m}}(t)=\beta_{\mathrm{m}}(\mathbf{p}) r(t)$, where $t \geq 0, r(t) \in \mathbb{R}^{m}$ is the reference-model command, and $y_{\mathrm{m}}(t) \in \mathbb{R}^{m}$ is the reference-model output.

Our objective is to design an output-feedback control such that the output trajectory $y$ follows the referencemodel trajectory $y_{\mathrm{m}}$, and thus, the error $z \triangleq y-y_{\mathrm{m}}$ is small. More specifically, we seek to make the average power $\mathcal{P}_{z} \triangleq \lim _{t \rightarrow \infty} \frac{1}{t} \int_{0}^{t} z^{\mathrm{T}}(\tau) z(\tau) \mathrm{d} \tau$ of the error small. In this study, we consider the pitch and altitude commandfollowing errors for a fixed-wing UAV.

If $\alpha_{\mathrm{m}}=\beta_{\mathrm{m}}$ and the reference model initial conditions are selected appropriately, then $y_{\mathrm{m}}=r$. In this case, $z=$ $y-r$, and the objective is to make $y$ follow $r$. In this study, we focus on reference-model dynamics with $\alpha_{\mathrm{m}}=\beta_{\mathrm{m}}$.

The ideal dynamic-inversion control is given by

$$
\begin{aligned}
& u_{*} \triangleq- H_{d}^{-1}\left[\alpha_{\mathrm{m}}(\mathbf{p}) y-\beta_{\mathrm{m}}(\mathbf{p}) r-\mathbf{p}^{d} y+C A^{d} x\right] \\
&-H_{d}^{-1}\left[\sum_{i=0}^{d-1} C A^{d-1-i} \mathbf{p}^{i} w\right],
\end{aligned}
$$

and it follows from [18, Lemma 1] that if $u=u_{*}$, then $\lim _{t \rightarrow \infty} z(t)=0$ and $\mathcal{P}_{z}=0$. Thus, the ideal dynamicinversion control $u_{*}$ accomplishes the control objective. However, Eq. (3) is not implementable, because $u_{*}$ depends on measurement of the full state $x$ and the disturbance $w$ as well as knowledge of $A, B$, and $C$.

Instead, we generate the control $u$ by passing $u_{*}$ through a low-pass filter. We consider the control $u$ that satisfies

$$
\eta_{k}(\mathbf{p}) u=\eta_{k}(0) u_{*},
$$

where $\eta_{k}(s)$ is a monic polynomial in $s$ with degree $\rho \geq d$ and real coefficients that are functions of a real parameter $k$. Define $\bar{\eta}_{k}(s) \triangleq\left(\eta_{k}(s)-\eta_{k}(0)\right) / s$. The polynomial $\eta_{k}$ is a design parameter, which is selected to satisfy the conditions:

(C1) There exists $k_{0}>0$, such that for all $k>k_{0}, \eta_{k}$ is Hurwitz.

(C2) For all $\epsilon>0$, there exists $k_{\epsilon}>k_{0}$ such that for all $k>k_{\epsilon}, \sup _{\omega \in \mathbb{R}}\left|\frac{\bar{\eta}_{k}(j \omega)}{\eta_{k}(j \omega)}\right|<\epsilon$.

See Ref. [18] for choices of $\eta_{k}$ that satisfy (C1) and (C2). For example, $\eta_{k}(s)=(s+k)^{\rho}$ satisfies $(\mathrm{C} 1)$ and $(\mathrm{C} 2)$.

The control $u$ cannot be implemented using Eq. (4) because $u_{*}$ depends on $x, w, A, B$, and $C$. To express $u$ as an implementable control, taking the $d$ th derivative of Eq. (2) and using Eq. (1) yields

$$
\mathbf{p}^{d} y=H_{d} u+C A^{d} x+\sum_{i=0}^{d-1} C A^{d-1-i} \mathbf{p}^{i} w .
$$

Substituting Eq. (5) into Eq. (3) implies that $u_{*}=u-$ $H_{d}^{-1}\left[\alpha_{\mathrm{m}}(\mathbf{p}) y-\beta_{\mathrm{m}}(\mathbf{p}) r\right]$, and combining with Eq. (4) yields the filtered-dynamic-inversion control

$$
\mathbf{p} \bar{\eta}_{k}(\mathbf{p}) u(t)=-\eta_{k}(0) H_{d}^{-1}\left[\alpha_{\mathrm{m}}(\mathbf{p}) y(t)-\beta_{\mathrm{m}}(\mathbf{p}) r(t)\right] .
$$

The FDI control is designed using the relative degree $d$, Markov parameter $H_{d}$, reference-model polynomials $\alpha_{\mathrm{m}}$ and $\beta_{\mathrm{m}}$, and the filter polynomial $\eta_{k}$, which depends on the real parameter $k$.

It follows from [18, Theorem 1] that, for sufficiently large $k>0$, the FDI control, given by Eq. (6), stabilizes the dynamic system, given by Eqs. (1) and (2), and makes $\mathcal{P}_{z}$ arbitrarily small. This paper uses FDI to improve the average power of the altitude command-following error for a small fixed-wing UAV in turbulent wind conditions.

\section{Nonlinear Dynamics for a Fixed-Wing UAV}

Let $F_{\mathrm{I}}$ be an inertial frame, that is, a frame in which Newton's second law is valid. Let $o_{\mathrm{I}}$ denote the center of $F_{\mathrm{I}}$, which has orthogonal unit vectors $\hat{\imath}_{\mathrm{I}}, \hat{\jmath}_{\mathrm{I}}$, and $\hat{k}_{\mathrm{I}}$. Let $F_{\mathrm{B}}$ denote the body frame, which is fixed to the UAV at its center of mass $o_{\mathrm{B}}$ and has orthogonal unit vectors $\hat{\imath}_{\mathrm{B}}$, $\hat{\jmath}_{\mathrm{B}}$, and $\hat{k}_{\mathrm{B}}$ as shown in Fig. 1 .

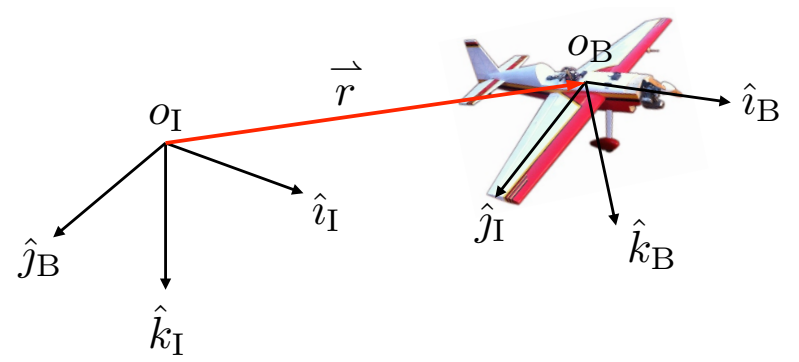

Figure 1: Inertial and Body Frames. The inertial frame $F_{\mathrm{I}}$ is centered at $o_{\mathrm{I}}$, and the body frame $F_{\mathrm{B}}$ is fixed to the UAV at its center of mass $o_{\mathrm{B}}$.

The position of the center of mass $o_{\mathrm{B}}$ relative to $o_{\mathrm{I}}$ is $\vec{r}=X \hat{\imath}_{\mathrm{I}}+Y \hat{\jmath}_{\mathrm{I}}+Z \hat{k}_{\mathrm{I}}$, and the velocity of $o_{\mathrm{B}}$ relative to $o_{\mathrm{I}}$ with respect to $F_{\mathrm{I}}$ is $\vec{v} \triangleq \mathrm{I} \cdot \vec{r}=\dot{X} \hat{\imath}_{\mathrm{I}}+\dot{Y} \hat{\jmath}_{\mathrm{I}}+\dot{Z} \hat{k}_{\mathrm{I}}$, where ${ }^{\mathrm{I} \cdot} \cdot \vec{r}$ is the time derivative of $\vec{r}$ with respect to $F_{\mathrm{I}}$. Let $\vec{\omega}$ be 
the angular velocity of $F_{\mathrm{B}}$ relative to $F_{\mathrm{I}}$. Let $[\cdot]_{\mathrm{B}}$ denote a physical vector resolved in the body frame $F_{\mathrm{B}}$. Thus, $\vec{v}$

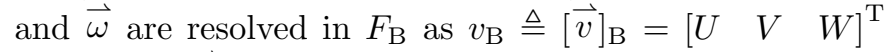
and $\omega_{\mathrm{B}} \triangleq[\vec{\omega}]_{\mathrm{B}}=\left[\begin{array}{lll}P & Q & R\end{array}\right]^{\mathrm{T}}$. Let $\phi, \theta$, and $\psi$ be the yaw, pitch, roll Euler angles defined by a 3-2-1 rotation sequence, which is standard in flight dynamics [21].

Let $m$ be the UAV's mass, and let $\vec{I}$ be the UAV's physical inertia matrix relative to the center of mass. We make the following assumptions:

(A1) Flight conditions are low speed and low altitude.

(A2) $m$ is constant.

(A3) The UAV is rigid.

(A4) The UAV is symmetric about the $\hat{\imath}_{\mathrm{B}}-\hat{k}_{\mathrm{B}}$ plane.

(A5) The thrust force acts through the center of mass.

(A6) The thrust force is in the $\hat{\imath}_{\mathrm{B}}-\hat{k}_{\mathrm{B}}$ plane.

Assumption (A1) implies that $o_{\mathrm{I}}$ can be any point on the Earth's surface and the curvature of the Earth can be neglected. Assumption (A2) implies that $\mathrm{d} m / \mathrm{d} t=0$, while (A2) and (A3) imply that $\mathrm{d}\left([\vec{I}]_{\mathrm{B}}\right) / \mathrm{d} t=0$. Assumption (A4) implies that $[\vec{I}]_{\mathrm{B}}$ can be expressed as

$$
[\vec{I}]_{\mathrm{B}}=\left[\begin{array}{ccc}
I_{\mathrm{xx}} & 0 & -I_{\mathrm{xz}} \\
0 & I_{\mathrm{yy}} & 0 \\
-I_{\mathrm{xz}} & 0 & I_{\mathrm{zz}}
\end{array}\right]
$$

where $I_{\mathrm{xx}}, I_{\mathrm{yy}}$, and $I_{\mathrm{zz}}$ are moments of inertia, and $I_{\mathrm{xz}}$ is a product of inertia. Assumption (A5) implies that the thrust force does not cause any moment about the center of mass, and (A6) implies that the thrust force in the $\hat{\jmath}_{\mathrm{B}}$ direction is zero.

Let $\vec{F}_{\mathrm{a}}$ be the aerodynamic force, and let $\vec{F}_{\mathrm{T}}$ be the thrust force, which are resolved as $\left[\vec{F}_{\mathrm{a}}\right]_{\mathrm{B}}=\left[X_{\mathrm{a}} Y_{\mathrm{a}} Z_{\mathrm{a}}\right]^{\mathrm{T}}$ and $\left[\begin{array}{ll}F_{\mathrm{T}}\end{array}\right]_{\mathrm{B}}=\left[\begin{array}{lll}X_{\mathrm{T}} & Y_{\mathrm{T}} & Z_{\mathrm{T}}\end{array}\right]^{\mathrm{T}}$, where $(\mathrm{A} 6)$ implies that $Y_{\mathrm{T}}=0$. Thus, Newton's second law in the body frame yields

$$
m \dot{v}_{\mathrm{B}}+m \Omega v_{\mathrm{B}}=m[\vec{g}]_{\mathrm{B}}+\left[\vec{F}_{\mathrm{a}}\right]_{\mathrm{B}}+\left[\overrightarrow{F_{\mathrm{T}}}\right]_{\mathrm{B}},
$$

where $\Omega \triangleq\left[\begin{array}{ccc}0 & -R & Q \\ R & 0 & -P \\ -Q & P & 0\end{array}\right]$ and $\vec{g}=g \hat{k}_{\mathrm{I}}$ is the acceleration due to gravity.

The moment about the UAV's center of mass due to the aerodynamic forces is denoted by $\vec{M}_{\mathrm{c}}$, which is resolved in $F_{\mathrm{B}}$ as $\left[\vec{M}_{\mathrm{c}}\right]_{\mathrm{B}}=[L M N]^{\mathrm{T}}$. Thus, Euler's equation yields

$$
\left[\vec{M}_{\mathrm{c}}\right]_{\mathrm{B}}=[\vec{I}]_{\mathrm{B}} \dot{\omega}_{\mathrm{B}}+\Omega[\vec{I}]_{\mathrm{B}} \omega_{\mathrm{B}} .
$$

The nonlinear equations of motion, given by Eqs. (7) and (8), depend on the aerodynamic forces $X_{\mathrm{a}}, Y_{\mathrm{a}}$, and $Z_{\text {a }}$, and aerodynamic moments $L, M$, and $N$. We now develop a nonlinear model of those forces and moments. Let $\overrightarrow{v_{\mathrm{w}}}$ denote the velocity of the wind, and define the relative velocity $\overrightarrow{v_{\mathrm{r}}} \triangleq \vec{v}-\overrightarrow{v_{\mathrm{w}}}$, which is resolved in $F_{\mathrm{B}}$ as $\left[\overrightarrow{v_{\mathrm{r}}}\right]_{\mathrm{B}}=$ $\left[\begin{array}{lll}U_{\mathrm{r}} & V_{\mathrm{r}} & W_{\mathrm{r}}\end{array}\right]^{\mathrm{T}}$. Define the airspeed $V_{\mathrm{T}} \triangleq \sqrt{U_{\mathrm{r}}^{2}+V_{\mathrm{r}}^{2}+W_{\mathrm{r}}^{2}}$, angle of attack $\alpha \triangleq \tan ^{-1} W_{\mathrm{r}} / U_{\mathrm{r}}$, and sideslip angle $\beta \triangleq$ $\sin ^{-1} V_{\mathrm{r}} / V_{\mathrm{T}}$. Let $\delta_{\mathrm{e}}, \delta_{\mathrm{r}}$, and $\delta_{\mathrm{a}}$ denote the angular deflections of the elevator, rudder, and ailerons, respectively.

The aerodynamic forces $X_{\mathrm{a}}$ and $Z_{\mathrm{a}}$, and the aerodynamic moment $M$ are each assumed to be functions of $V_{\mathrm{T}}$, $\alpha, \beta, Q, \delta_{\mathrm{e}}, \delta_{\mathrm{r}}$, and $\delta_{\mathrm{a}}$. Similarly, $Z_{\mathrm{a}}, L$, and $N$ are assumed to be functions of $V_{\mathrm{T}}, \alpha, \beta, P, R, \delta_{\mathrm{e}}, \delta_{\mathrm{r}}$, and $\delta_{\mathrm{a}}$. In this paper, the aerodynamic forces and moments do not depend on $\dot{\alpha}$ or $\dot{\beta}$, because these dependencies, which cannot be estimated using steady-state fluid dynamic analysis, are assumed to be negligible.

We use Taylor-series expansions to approximate $X_{\mathrm{a}}$, $Y_{\mathrm{a}}, Z_{\mathrm{a}}, L, M$, and $N$ in a neighborhood of $\omega_{\mathrm{B}}=0$ and $\delta_{\mathrm{e}}=$ $\delta_{\mathrm{r}}=\delta_{\mathrm{a}}=0$. For example, we approximate $X_{\mathrm{a}}$ as $X_{\mathrm{a}}=$ $\left(X_{\mathrm{a}}\right)_{0}+\left(\partial X_{\mathrm{a}} / \partial Q\right)_{0} Q+\left(\partial X_{\mathrm{a}} / \partial \delta_{\mathrm{e}}\right)_{0} \delta_{\mathrm{e}}+\left(\partial X_{\mathrm{a}} / \partial \delta_{\mathrm{r}}\right)_{0} \delta_{\mathrm{r}}+$ $\left(\partial X_{\mathrm{a}} / \partial \delta_{\mathrm{a}}\right)_{0} \delta_{\mathrm{a}}$, where $(\cdot)_{0}$ means that the function is evaluate at $\left(P, Q, R, \delta_{\mathrm{e}}, \delta_{\mathrm{r}}, \delta_{\mathrm{a}}\right)=0$. Each term in each Taylorexpansion is an explicit function of $V_{\mathrm{T}}, \alpha$, and $\beta$.

For the UAV in this study, the functional approximations for the aerodynamic forces and moments $X_{\mathrm{a}}, Y_{\mathrm{a}}, Z_{\mathrm{a}}$, $L, M$, and $N$ are estimated using Athena Vortex Lattice (AVL), which is a vortex lattice aerodynamics solver. For example, $X_{\mathrm{a}}$ depends on the functions $\left(X_{\mathrm{a}}\right)_{0},\left(\partial X_{\mathrm{a}} / \partial Q\right)_{0}$, $\left(\partial X_{\mathrm{a}} / \partial \delta_{\mathrm{e}}\right)_{0},\left(\partial X_{\mathrm{a}} / \partial \delta_{\mathrm{r}}\right)_{0}$, and $\left(\partial X_{\mathrm{a}} / \partial \delta_{\mathrm{a}}\right)_{0}$. The value of each function is estimated for 524 values of $\left(V_{\mathrm{T}}, \alpha, \beta\right)$. Specifically, the value of each function is estimated for $\left(V_{\mathrm{T}}, \alpha, \beta\right) \in\{10,15,20,25,30,35\} \times\left\{-\frac{\pi}{6},-\frac{\pi}{9},-\frac{\pi}{15},-\frac{\pi}{30}\right.$, $\left.-\frac{\pi}{45},-\frac{\pi}{90}, 0, \frac{\pi}{90}, \frac{\pi}{45}, \frac{\pi}{30}, \frac{\pi}{15}, \frac{\pi}{9}, \frac{\pi}{6}\right\} \times\left\{-\frac{\pi}{6},-\frac{\pi}{15},-\frac{\pi}{45}, 0, \frac{\pi}{45}, \frac{\pi}{15}\right.$, $\left.\frac{\pi}{6}\right\}$, where $V_{\mathrm{T}}$ is in $\mathrm{m} / \mathrm{s}, \alpha$ is in $\mathrm{rad}$, and $\beta$ is in $\mathrm{rad}$. Then, each function is approximated using linear interpolation between the 524 values.

\section{FDI Control System}

Consider the guidance and control system shown in Fig. 2, which uses mission information, GPS measurements, and speed command $U_{\mathrm{d}}$ as well as feedback of the pitch $\theta$, speed $U_{\mathrm{r}}$, and altitude $h \triangleq-Z$ to generate throttle command $u_{\mathrm{T}}$ and elevator command $u_{\mathrm{e}}$. The guidance system uses mission information and GPS measurements to generate the altitude command $h_{\mathrm{d}}$. The outer-loop altitude controller uses the command $h_{\mathrm{d}}$ and feedback $h$ to generate the pitch command $\theta_{\mathrm{d}}$. The speed controller uses the speed command $U_{\mathrm{d}}$ and feedback $U_{\mathrm{r}}$ to generate the throttle command $u_{\mathrm{T}}$, while the pitch controller uses $\theta_{\mathrm{d}}$ and the feedback $\theta$ to generate the elevator command $u_{\mathrm{e}}$.

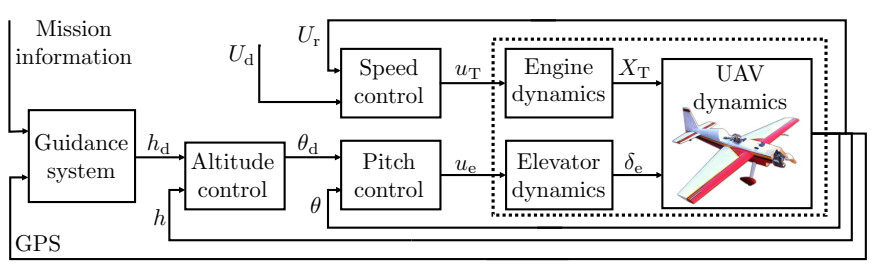

Figure 2: Guidance and Control System. The altitude, pitch, and speed controllers use feedback of the altitude $h$, pitch $\theta$, and speed $U_{\mathrm{r}}$ to generate the throttle command $u_{\mathrm{T}}$ and elevator command $u_{\mathrm{e}}$.

Our control objective is altitude command following in the presence of an unknown and potentially turbulent wind. Let $\mathcal{P}_{h}\left(t_{1}, t_{0}\right)$ denote the average power of altitude error over the time interval $\left[t_{0}, t_{1}\right)$, that is,

$$
\mathcal{P}_{h}\left(t_{1}, t_{0}\right) \triangleq \frac{1}{t_{1}-t_{0}} \int_{t_{0}}^{t_{1}}\left[h_{\mathrm{d}}(\tau)-h(\tau)\right]^{2} \mathrm{~d} \tau .
$$


We propose reducing $\mathcal{P}_{h}\left(t_{1}, t_{0}\right)$ by implementing FDI in the multi-loop control system in Fig. 2. However, the linearized UAV transfer function from elevator command $u_{\mathrm{e}}$ to altitude $h$ is nonminimum phase. Thus, FDI cannot be used to control altitude $h$ directly. Instead, the pitch error $\theta_{\mathrm{d}}-\theta$ is used as a surrogate for altitude error $h_{\mathrm{d}}-h$. As shown in the next section, the linearized transfer function from $u_{\mathrm{e}}$ to $\theta$ is minimum phase. Consider the average power of the pitch error over the time interval $\left[t_{0}, t_{1}\right)$, which is

$$
\mathcal{P}_{\theta}\left(t_{1}, t_{0}\right) \triangleq \frac{1}{t_{1}-t_{0}} \int_{t_{0}}^{t_{1}}\left[\theta_{\mathrm{d}}(\tau)-\theta(\tau)\right]^{2} \mathrm{~d} \tau .
$$

Since the UAV's linearized transfer function from $u_{\mathrm{e}}$ to $\theta$ is minimum phase, the main result of Ref. [18] suggests that FDI can be used to make $\lim _{t_{1} \rightarrow \infty} \mathcal{P}_{\theta}\left(t_{1}, t_{0}\right)$ arbitrarily small.

We present an FDI flight-control system, where innerloop pitch control uses FDI, while inner-loop speed control and outer-loop altitude control uses proportional-integral (PI) control.

\section{Altitude Control}

An altitude error outer-loop controller is used to generate the pitch command $\theta_{\mathrm{d}}$. Consider the PI controller

$$
\theta_{\mathrm{d}}(t)=k_{h, \mathrm{p}}\left[h_{\mathrm{d}}(t)-h(t)\right]+k_{h, \mathrm{i}} \int_{0}^{t}\left[h_{\mathrm{d}}(\tau)-h(\tau)\right] \mathrm{d} \tau,
$$

where $k_{h, \mathrm{p}} \in \mathbb{R}$ is that proportional gain and $k_{h, \mathrm{i}} \in \mathbb{R}$ is the integral gain.

\section{Speed Control}

The thrust force is assumed to act only in the $\hat{\imath}_{\mathrm{B}}$ direction, which implies that $Z_{\mathrm{T}}=0$. The engine dynamics are assumed to be negligible. Specifically, the thrust force $X_{\mathrm{T}}$ is proportional to throttle command $u_{\mathrm{T}}$, that is, $X_{\mathrm{T}}=g_{\mathrm{T}} u_{\mathrm{T}}$, where $g_{\mathrm{T}} \in \mathbb{R}$ is the engine gain. For speed control, consider the PI controller

$X_{\mathrm{T}}(t)=k_{\mathrm{T}, \mathrm{p}}\left[U_{\mathrm{d}}(t)-U_{\mathrm{r}}(t)\right]+k_{\mathrm{T}, \mathrm{i}} \int_{0}^{t}\left[U_{\mathrm{d}}(\tau)-U_{\mathrm{r}}(\tau)\right] \mathrm{d} \tau$,

where $k_{\mathrm{T}, \mathrm{p}} \in \mathbb{R}$ is the proportional gain and $k_{\mathrm{T}, \mathrm{i}} \in \mathbb{R}$ is the integral gain.

\section{Pitch Control}

FDI is implemented as the pitch controller. Recall from Section 3 that FDI requires limited model information, specifically, the relative degree $d$ and Markov parameter $H_{\mathrm{d}}$ of the linearized transfer function from the control $u_{\mathrm{e}}$ to the pitch $\theta$. FDI also requires that the linearized transfer function from $u_{\mathrm{e}}$ to $\theta$ is minimum phase. To examine these requirements, Eqs. (7) and (8) are linearized about a constant-velocity, constant-altitude, wings-level flight condition. In this case, $\phi=V=P=R=L=N=Y_{\mathrm{a}}=0$. We also assume that there is no wind, that is, $\overrightarrow{v_{\mathrm{w}}}=0$.
Let $U_{0}, W_{0}, \theta_{0}, X_{\mathrm{T}, 0}$, and $\delta_{\mathrm{e}, 0}$ denote the equilibrium $\hat{\imath}_{\mathrm{B}}$-direction velocity, $\hat{k}_{\mathrm{B}}$-direction velocity, pitch, thrust, and elevator deflection used in the linearization. Define the perturbation variables $\Delta U(t) \triangleq U(t)-U_{0}, \Delta W(t) \triangleq$ $W(t)-W_{0}, \Delta \theta(t) \triangleq \theta(t)-\theta_{0}, \Delta X_{\mathrm{T}}(t) \triangleq X_{\mathrm{T}}(t)-X_{\mathrm{T}, 0}$, and $\Delta \delta_{\mathrm{e}}(t) \triangleq \delta_{\mathrm{e}}(t)-\delta_{\mathrm{e}, 0}$. Using the standard aircraft linearization process [21], we obtain the linearized equations of motion for longitudinal flight, which are

$$
\dot{x}_{1}(t)=A_{\mathrm{l}} x_{\mathrm{l}}(t)+B_{\mathrm{l}, \delta_{\mathrm{e}}} \Delta \delta_{\mathrm{e}}(t)+B_{\mathrm{l}, X_{\mathrm{T}}} \Delta X_{\mathrm{T}}(t),
$$

where $x_{1}(t) \triangleq\left[\begin{array}{llll}\Delta U(t) & \Delta W(t) & Q(t) & \Delta \theta(t)\end{array}\right]^{\mathrm{T}}$, and

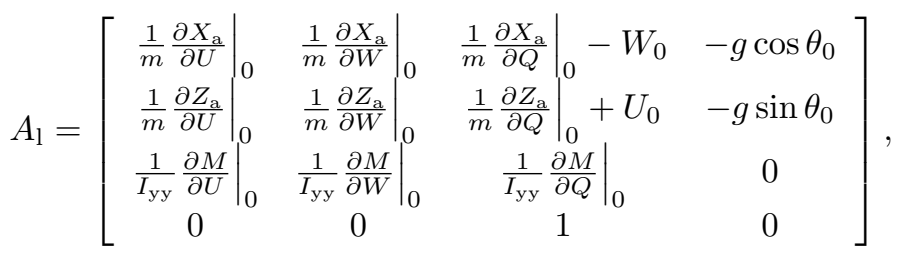

$$
\begin{aligned}
& B_{1, \delta_{\mathrm{e}}} \triangleq\left[\begin{array}{c}
\left.\frac{1}{m} \frac{\partial X_{\mathrm{a}}}{\partial \delta_{\mathrm{e}}}\right|_{0} \\
\left.\frac{1}{m} \frac{\partial Z_{\mathrm{a}}}{\partial \delta_{\mathrm{e}}}\right|_{0} \\
\left.\frac{1}{I_{\mathrm{yy}}} \frac{\partial M}{\partial \delta_{\mathrm{e}}}\right|_{0} \\
0
\end{array}\right], \quad B_{1, X_{\mathrm{T}}} \triangleq\left[\begin{array}{c}
\frac{1}{m} \\
0 \\
0 \\
0
\end{array}\right]
\end{aligned}
$$

where the subscript zero indicates evaluating at the forced equilibrium.

Define $\Delta U_{\mathrm{d}}(t) \triangleq U_{\mathrm{d}}(t)-U_{0}$, and cascading the speed control (10) with the longitudinal dynamics (11) yields

$$
\dot{\chi}(t)=\mathcal{A} \chi(t)+\mathcal{B}_{\delta_{\mathrm{e}}} \Delta \delta_{\mathrm{e}}(t)+\mathcal{B}_{U_{\mathrm{d}}} \Delta U_{\mathrm{d}}(t),
$$

where $\chi(t) \triangleq\left[\begin{array}{ll}x_{1}^{\mathrm{T}}(t) & x_{\mathrm{T}, \mathrm{i}}(t)\end{array}\right]^{\mathrm{T}}, x_{\mathrm{T}, \mathrm{i}}(t) \in \mathbb{R}$ is the integrator state of Eq. (10), and

$$
\begin{gathered}
\mathcal{A} \triangleq\left[\begin{array}{cc}
A_{\mathrm{l}}-m k_{\mathrm{T}, \mathrm{p}} B_{1, X_{\mathrm{T}}} B_{\mathrm{l}, X_{\mathrm{T}}}^{\mathrm{T}} & k_{\mathrm{T}, \mathrm{i}} B_{1, X_{\mathrm{T}}} \\
-m B_{\mathrm{l}, X_{\mathrm{T}}}^{\mathrm{T}} & 0
\end{array}\right], \\
\mathcal{B}_{\delta_{\mathrm{e}}} \triangleq\left[\begin{array}{c}
B_{1, \delta_{\mathrm{e}}} \\
0
\end{array}\right], \quad \mathcal{B}_{U_{\mathrm{d}}} \triangleq\left[\begin{array}{c}
B_{1, X_{\mathrm{T}}} \\
1
\end{array}\right] .
\end{gathered}
$$

The pitch perturbation is given by $\Delta \theta(t)=\mathcal{C}_{\theta} \chi(t)$, where $\mathcal{C}_{\theta} \triangleq\left[\begin{array}{lllll}0 & 0 & 0 & 1 & 0\end{array}\right]$. Thus, the linearized transfer function from $\Delta \delta_{\mathrm{e}}$ to $\Delta \theta$ is $\mathrm{C}_{\theta}(s I-\mathcal{A})^{-1} \mathcal{B}_{\delta_{\mathrm{e}}}$.

The elevator actuator dynamics are modeled by $\tau_{\mathrm{e}} \dot{\delta}_{\mathrm{e}}(t)+$ $\delta_{\mathrm{e}}(t)=u_{\mathrm{e}}(t)$, where $\tau_{\mathrm{e}}>0$ is the time constant associated with the elevator servomechanism. Define the elevator command perturbation $\Delta u_{\mathrm{e}}(t) \triangleq u_{\mathrm{e}}(t)-\delta_{\mathrm{e}, 0}$, and it follows that the elevator actuator dynamics are

$$
\tau_{\mathrm{e}} \Delta \dot{\delta}_{\mathrm{e}}(t)+\Delta \delta_{\mathrm{e}}(t)=\Delta u_{\mathrm{e}}(t)
$$

Therefore, Eqs. (12)-(15) imply that the linearized transfer function from $\Delta u_{\mathrm{e}}$ to $\Delta \theta$ is

$$
\begin{aligned}
G(s) & \triangleq \frac{1 / \tau_{\mathrm{e}}}{s+1 / \tau_{\mathrm{e}}} \mathcal{C}_{\theta}(s I-\mathcal{A})^{-1} \mathcal{B}_{\delta_{\mathrm{e}}} \\
& =\frac{b_{3} s^{3}+b_{2} s^{2}+b_{1} s+b_{0}}{s^{6}+a_{5} s^{5}+a_{4} s^{4}+a_{3} s^{3}+a_{2} s^{2}+a_{1} s+a_{0}},
\end{aligned}
$$

where $a_{0}, \ldots, a_{5}, b_{0}, \ldots, b_{3} \in \mathbb{R}$. The Routh stability criteria imply that $G$ is minimum phase if and only if $b_{0}, b_{1}, b_{2}, b_{3}$ have the same sign and $b_{1} b_{2}-b_{0} b_{3}>0$. 
For the small fixed-winged UAV considered in this paper, the numerator coefficients $b_{0}, b_{1}, b_{2}, b_{3}$ are all negative and $b_{1} b_{2}-b_{0} b_{3}>0$, which implies that $G$ is minimum phase. Since the relative degree is $d=3$, we consider the FDI controller (6) with $m=1, \alpha_{\mathrm{m}}=\beta_{\mathrm{m}}$, and $\rho \geq d=3$. In this case, Eq. (6) becomes

$$
\mathbf{p} \bar{\eta}_{k}(\mathbf{p}) \Delta u_{\mathrm{e}}(t)=\frac{\eta_{k}(0)}{H_{d}} \alpha_{\mathrm{m}}(\mathbf{p})\left[\theta_{\mathrm{d}}(t)-\theta(t)\right]
$$

where the degree of $\alpha_{\mathrm{m}}$ is 3, and Eqs. (12)-(15) imply that the first nonzero Markov parameter is $H_{d}=b_{3}=$ $\left.\frac{1}{I_{\mathrm{yy}} \tau_{\mathrm{e}}} \frac{\partial M}{\partial \delta_{\mathrm{e}}}\right|_{0}$. Taking the Laplace transform of Eq. (17) yields the FDI controller transfer function

$$
C_{k}(s) \triangleq \frac{\eta_{k}(0) \alpha_{\mathrm{m}}(s)}{H_{\mathrm{d}} \bar{\eta}_{k}(s) s},
$$

where the subscript $k$ denotes the dependence on the FDI parameter $k$. The linearized closed-loop transfer function from pitch command to pitch error is $\tilde{G}_{k} \triangleq 1 /\left(1+G C_{k}\right)$. It follows from [18, Theorem 1] that, for sufficiently large $k>0, \tilde{G}_{k}$ is asymptotically stable and the average power of the pitch error $\lim _{t_{1} \rightarrow \infty} \mathcal{P}_{\theta}\left(t_{1}, t_{0}\right)$ is arbitrarily small.

\section{FDI Design for a Small Fixed-Wing UAV}

\subsection{Description of the $U A V$}

We constructed a fixed-wing UAV using the AeroWorks EDGE 540T mid-winged remote-controlled aircraft. The airframe had an unswept, tapered wing with span $b_{\mathrm{r}}=1.52$ $\mathrm{m}$, mean chord length $c_{\mathrm{r}}=0.2975 \mathrm{~m}$, and planform area $S_{\mathrm{r}}=0.4534 \mathrm{~m}^{2}$. The leading edge of the horizontal stabilizer was located $0.806 \mathrm{~m}$ from the leading edge of the wing. The fully loaded aircraft had mass $m=4.48 \mathrm{~kg}$ at takeoff with the center of gravity located $70 \mathrm{~mm}$ aft of the wing's leading edge, in accordance with the manufacturer's specifications. For propulsion, the UAV used an Electrifly RimFire 0.80 brushless outboard electric motor, which was rated for $1300 \mathrm{~W}$ constant output. The motor was mounted along the $\hat{\imath}_{\mathrm{B}}$ axis. The electric motor was powered by two $8 \mathrm{~S}$ lithium-polymer batteries wired in series, each with capacity $5000 \mathrm{mAh}$. This setup was capable of nine minutes of flight. The principle moments of inertia were measured using the experimental procedure in [22]; they were $I_{\mathrm{xx}}=0.1778 \mathrm{~kg} \cdot \mathrm{m}^{2}, I_{\mathrm{yy}}=0.3287 \mathrm{~kg} \cdot \mathrm{m}^{2}$, and $I_{\mathrm{zz}}=0.4231 \mathrm{~kg} \cdot \mathrm{m}^{2}$. Aerodynamic coefficients for the airframe were estimated using AVL (as described in Section 4 ), and the values are available in [23].

The low-rate control deflections recommended by the manufacturer were used during flight. Thus, the elevator was limited to a $15^{\circ}$ deflection. The elevator servo was a Hitec HS-645MG High-Torque 2BB Metal Gear servo, which had a maximum angular velocity of $300^{\circ} / \mathrm{s}$.

\subsection{FDI Control System Design}

The altitude and speed control parameters $k_{h, \mathrm{p}}, k_{h, \mathrm{i}}$, $k_{\mathrm{T}, \mathrm{p}}$, and $k_{\mathrm{T}, \mathrm{i}}$ are designed using the longitudinal UAV dynamics linearized about a constant-velocity, constantaltitude, wings-level flight condition, where $U_{0}=19.9 \mathrm{~m} / \mathrm{s}$,
$W_{0}=1.95 \mathrm{~m} / \mathrm{s}, \theta_{0}=5.6^{\circ}$, and $\delta_{\mathrm{e}, 0}=-3.94^{\circ}$. Using simulation-based tuning, the PI controller parameters are selected as $k_{h, \mathrm{p}}=1.6, k_{h, \mathrm{i}}=0.2, k_{\mathrm{T}, \mathrm{p}}=2$, and $k_{\mathrm{T}, \mathrm{i}}=0.5$.

We use the linearized transfer function (16) to design the FDI controller (17). To obtain a model of the linearized transfer function (16), we require estimates of the aerodynamic coefficients in Eq. (11), and an estimate of the elevator servomechanism time constant $\tau_{\mathrm{e}}$. The aerodynamic coefficients are estimated using AVL. Next, we note that the elevator servomechanism has approximately $60^{\circ}$ fullstroke angle (i.e., $\pm 30^{\circ}$ ). The servomechanism time constant $\tau_{\mathrm{e}}$ is approximated by assuming that the full-stroke (i.e., $60^{\circ}$ ) step response of the servomechanism reaches but does not exceed the servomechanism's maximum angular velocity of $300^{\circ} / \mathrm{s}$. It follows from Eq. (15) that if $\Delta u_{\mathrm{e}}$ is the $60^{\circ}$-step function, then $\Delta \delta_{\mathrm{e}}(t)=60-60 e^{-t / \tau_{\mathrm{e}}} \operatorname{deg}$. Thus, the rate of $\Delta \delta_{\mathrm{e}}(t)$ reaches a maximum at $t=0^{+}$, and $\Delta \dot{\delta_{\mathrm{e}}}\left(0^{+}\right)=60 / \tau_{\mathrm{e}}$. To approximate $\tau_{\mathrm{e}}$, we let $60 / \tau_{\mathrm{e}}=$ $300 \mathrm{deg} / \mathrm{s}$, or, equivalently, $\tau_{\mathrm{e}}=0.2 \mathrm{~s}$. Note that the elevator dynamics for full-scale aircraft are often assumed to be first order with a time constant of $0.1 \mathrm{~s}$ [24, p. 59]. In this study, the elevator dynamics are assumed to be slower with time constant $\tau_{\mathrm{e}}=0.2 \mathrm{~s}$.

Together, the aerodynamic coefficients from AVL and the time constant $\tau_{\mathrm{e}}$ yield the linearized transfer function $G$, where $b_{3}=-636.6, b_{2}=-3,472, b_{1}=-1,729, b_{0}=$ $-355.7, a_{5}=17.29, a_{4}=188.0, a_{3}=690.4, a_{2}=321.9$, $a_{1}=170.5$, and $a_{0}=-2.480$. The zeros of $G$ are approximately -4.93 and $-0.264 \pm \jmath 0.209$, where $\jmath=\sqrt{-1}$. The zeros of $G$ are in the open-left-half complex plane, which verifies that $G$ is minimum phase.

The FDI controller (17) requires knowledge of the relative degree $d=3$ and the first nonzero Markov parameter $H_{d}$. No other UAV model information is required in order to design the FDI controller. The Markov parameter $H_{d}$ is estimated using

$$
H_{d}=\left.\frac{1}{I_{\mathrm{yy}} \tau_{\mathrm{e}}} \frac{\partial M}{\partial \delta_{\mathrm{e}}}\right|_{0} \approx \frac{\rho_{\mathrm{a}} V_{\mathrm{T}}^{2} S_{\mathrm{r}} c_{\mathrm{r}}\left(\left.C M_{\delta_{\mathrm{e}}}\right|_{0}\right)}{2 I_{\mathrm{yy}} \tau_{\mathrm{e}}},
$$

where $\rho_{\mathrm{a}}=1.22 \mathrm{~kg} / \mathrm{m}^{3}$ is air density, and $C M_{\delta_{\mathrm{e}}}$ is the derivative of dimensionless pitching moment coefficient with respect to elevator angle, which can be estimated using wind tunnel data or computational fluid dynamics software. We compute $H_{d}$ using Eq. (18), with measured values for $S_{\mathrm{r}}, c_{\mathrm{r}}$, and $I_{\mathrm{yy}}$, and the AVL estimate for $\left.C M_{\delta_{\mathrm{e}}}\right|_{0}$. In general, $V_{\mathrm{T}}$ is a function of time. However, [18, Corollary 1] demonstrates that FDI is robust to uncertainty in $H_{d}$. The assumption that $H_{d}$ is known can be replaced by the assumption that the sign of $H_{d}$ is known and an upper bound $\bar{H}_{d}$ on the magnitude of $H_{d}$ is known. In this case, $H_{d}$ in Eq. (17) is replaced by $\operatorname{sgn}\left(H_{d}\right) \bar{H}_{d}$. Although the numerical and physical experiments are performed at $V_{\mathrm{T}}=20 \mathrm{~m} / \mathrm{s}$, we compute the upper bound $\bar{H}_{d}$ using Eq. (18) with $V_{\mathrm{T}}=25 \mathrm{~m} / \mathrm{s}$. In this case, we obtain $\operatorname{sgn}\left(H_{d}\right)=-1$ and $\bar{H}_{d}=1005$.

To design the FDI controller, we select the controller order $\rho$, the filter polynomial $\eta_{k}$, and the reference-model polynomial $\alpha_{\mathrm{m}}$. Selecting $\rho=d+1$ yields the lowest order strictly proper controller, that is, a controller that 
does not include direct feed through of the feedback signal. Numerical testing suggests that the minimum stabilizing value of the parameter $k$ with $\rho=d+1$ tends to be smaller than the minimum stabilizing value of the parameter $k$ with $\rho>d+1$. Thus, we select $\rho=d+1=4$.

The filter polynomial $\eta_{k}$ is selected to have degree $\rho$ and satisfy $(\mathrm{C} 1)$ and $(\mathrm{C} 2)$. We let $\eta_{k}(s)=(s+k)^{4}$. Other choices of $\eta_{k}$ are given in Ref. [18].

The reference-model polynomial $\alpha_{\mathrm{m}}$ can be interpreted as the desired closed-loop dynamics for the pitch error. Specifically, the roots of $\alpha_{\mathrm{m}}$ are target pole locations for the closed-loop transfer function $\tilde{G}_{k} \triangleq 1 /\left(1+G C_{k}\right)$. In fact, as $k \rightarrow \infty, d$ closed-loop poles tend toward the roots of $\alpha_{\mathrm{m}}$, while the remaining closed-loop poles either tend toward the open-loop zeros or diverge to infinity through the open-left-half complex plane [19]. In this case, 3 poles of $\tilde{G}_{k}$ tend toward the roots of $\alpha_{\mathrm{m}}, 3$ poles of $\tilde{G}_{k}$ tend toward the zeros of $G$, and the remaining 4 poles of $\tilde{G}_{k}$ diverge through the open-left-half complex plane. Since $\alpha_{\mathrm{m}}$ is Hurwitz and $G$ is minimum phase, it follows that $\tilde{G}_{k}$ is asymptotically stable for sufficiently large $k$.

Since the roots of $\alpha_{\mathrm{m}}$ represent target closed-loop pole locations, it is often desirable to design each root of $\alpha_{\mathrm{m}}$ to be heavily damped (i.e., the ratio of the real part to the imaginary part is large). Physical limitations should also be taken into consideration for the design of $\alpha_{\mathrm{m}}$. For example, it is not practical for the roots of $\alpha_{\mathrm{m}}$ to have large magnitude relative to the magnitude of the openloop poles. Since closed-loop poles tend toward the roots of $\alpha_{\mathrm{m}}$, it follows that forcing closed-loop poles to have large magnitude might require prohibitively large elevator deflections $\delta_{\mathrm{e}}$. Every open-loop pole of $G$ has magnitude less than $11 \mathrm{rad} / \mathrm{s}$. Thus, we select $\alpha_{\mathrm{m}}$ such that each root is heavily damped (specifically, real) and has magnitude less than $11 \mathrm{rad} / \mathrm{s}$. Specifically, we let $\alpha_{\mathrm{m}}(s)=(s+4)(s+$ $6)(s+8)$. In this case, the FDI controller (17) becomes

$$
\left[\mathbf{p}^{4}+4 k \mathbf{p}^{3}+6 k^{2} \mathbf{p}^{2}+4 k \mathbf{p}\right] \Delta u_{\mathrm{e}}(t)=-\frac{k^{4}}{\bar{H}_{d}} \alpha_{\mathrm{m}}(\mathbf{p}) \tilde{\theta}(t),
$$

where $\tilde{\theta}(t) \triangleq \theta_{\mathrm{d}}(t)-\theta(t)$ is the pitch error and $k$ is positive. It can be shown from direct computation that the linearized closed-loop transfer function $\tilde{G}_{k}$ is asymptotically stable for all $k \geq 12$.

\subsection{Baseline Pitch Control}

We also design a PI controller, which is a standard flight controller, to evaluate the relative performance of FDI. This PI pitch controller is

$$
\Delta u_{\mathrm{e}}(t)=-k_{\theta, \mathrm{p}}\left[\theta_{\mathrm{d}}(t)-\theta(t)\right]+k_{\theta, \mathrm{i}} \int_{0}^{t}\left[\theta_{\mathrm{d}}(\tau)-\theta(\tau)\right] \mathrm{d} \tau
$$

where $k_{\theta, \mathrm{p}}, k_{\theta, \mathrm{i}} \in \mathbb{R}$. Note that the linearized longitudinal transfer function $G$ is relative degree 3 , and classical root locus for a relative degree 3 system shows that at least one closed-loop pole diverges into the open-right-half complex plane as the magnitude of the gain $k_{\theta, \mathrm{p}}$ is increased. Thus, the gain $k_{\theta, \mathrm{p}}$ cannot be made arbitrarily large without destabilizing the closed-loop dynamics.
We use root locus to examine potential designs of the PI controller gain $k_{\theta, \mathrm{p}}$ and the controller zero location $k_{\theta, \mathrm{i}} / k_{\theta, \mathrm{p}}$. For any choice of the zero $k_{\theta, \mathrm{i}} / k_{\theta, \mathrm{p}}$, the closedloop dynamics are unstable if $k_{\theta, \mathrm{p}}$ is larger than approximately 2.25. For example, assume $k_{\theta, \mathrm{i}} / k_{\theta, \mathrm{p}}=-0.2$. Then, for all $k_{\theta, \mathrm{p}} \in(0.005,2.2)$, every closed-loop pole is in the open-left-half complex plane, and the closed-loop dynamics are asymptotically stable. However, for all $k_{\theta, \mathrm{p}}>2.2$, at least one closed-loop pole is in the open-right-half complex plane, and the closed-loop dynamics are unstable. The closed-loop dynamics are also unstable for all $k_{\theta, \mathrm{p}}<0$.

The gains $k_{\theta, \mathrm{p}}=0.5$ and $k_{\theta, \mathrm{i}}=-0.1$ were designed through numerical testing. These gains provide the best closed-loop pitch command following of all designs considered. While the gain $k_{\theta, \mathrm{p}}$ can be increased up to 2.2 before destabilizing the closed-loop dynamics, values of $k_{\theta, \mathrm{p}}$ larger than 0.5 result in worse behavior because the closedloop transfer function has a pair of lightly damped poles.

We used the linearized transfer function to determine stabilizing values of $k_{\theta, \mathrm{p}}$ and $k_{\theta, \mathrm{i}}$. Then, we used numerical testing with the nonlinear model to determine values that yielded the best performance. In contrast, the FDI controller design relied on knowledge of only $d$ and $H_{\mathrm{d}}$.

Figure 3 shows the Bode plot of the PI pitch controller (20) and the Bode plot of FDI pitch controller (19) for different values of $k$. Recall that the closed-loop dynamics with the FDI controller are asymptotically stable for all $k \geq 12$, and notice that increasing $k$ increases the magnitude of the FDI controller across all frequencies. Thus, increasing $k$ tends to reduce the magnitude of the $\tilde{G}_{k}$, which, in turn, tends to improve pitch command following. In contrast to the FDI parameter $k$, the PI controller gain $k_{\theta, \mathrm{p}}$ cannot be made arbitrarily large without destabilizing the closed-loop transfer function. Thus, the magnitude of the PI controller cannot be increased to improve pitch command following. Figure 3 shows that for $k \geq 25$, the magnitude of the FDI controller is larger than the magnitude of the PI controller across all frequencies.
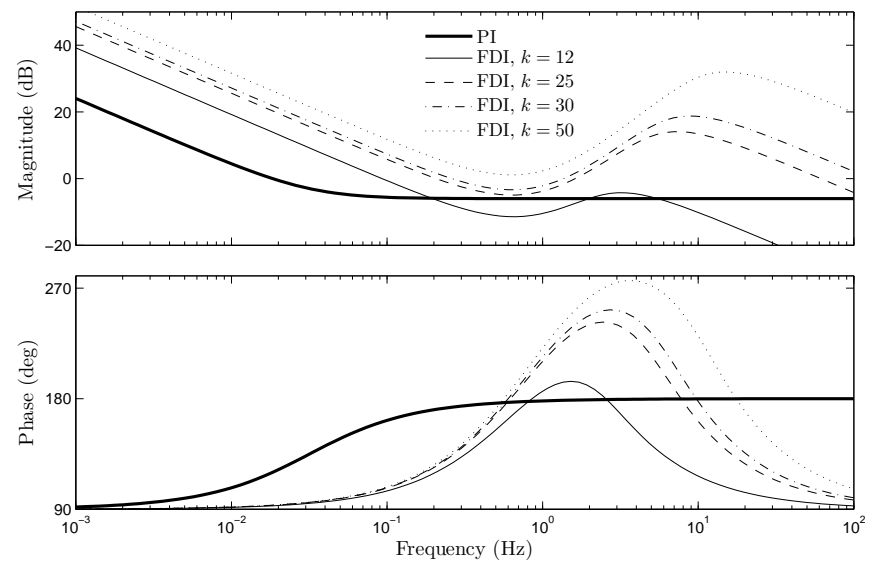

Figure 3: Bode Plots of the PI and FDI Pitch Controllers. Both the PI and FDI controllers have integral action. The magnitude of the FDI controller increases across all frequencies as $k$ is increases. 


\section{Closed-Loop Numerical Simulations}

This section presents numerical simulations of the nonlinear UAV model (7) and (8), where the aerodynamic parameters are estimated using AVL. We implement the altitude, speed, and pitch control system from the previous section. The performance of the FDI pitch controller (19) is compared to the performance of the PI pitch controller (20). All simulations use the physical UAV parameters given in Section 6.1. Since the UAV is a mid-winged aircraft that is approximately symmetric about the $\hat{\imath}_{\mathrm{B}}-\hat{\jmath}_{\mathrm{B}}$ plane, it follows that the product of inertia $I_{\mathrm{xz}}$ is small relative to the moments of inertia $I_{\mathrm{xx}}, I_{\mathrm{yy}}$, and $I_{\mathrm{zz}}$. For simplicity, we assume $I_{\mathrm{xz}}=0$.

To maintain wings-level flight, we consider the rollto-aileron PI controller $\delta_{\mathrm{a}}(t)=k_{\phi, \mathrm{p}} \phi(t)+k_{\phi, \mathrm{i}} \int_{0}^{t} \phi(\tau) \mathrm{d} \tau$, where $k_{\phi, \mathrm{p}}=0.5$ and $k_{\phi, \mathrm{i}}=1$. The roll control allows us to focus on the longitudinal dynamics. For all simulations, the initial heading is $\psi(0)=0$, and the heading is nearly constant, that is, $\psi(t) \approx 0$ for all $t \geq 0$.

Two 3-dimensional stochastic realizations are used to model the wind in the inertial frame $F_{\mathrm{I}}$. The first wind model is band-limited, zero-mean, unit-variance Gaussian white noise. The second wind model is an approximation for turbulence. This approximation is a zero-mean, unitvariance Gaussian random sequence whose power spectrum is filtered to decay in wavenumber space with a $-5 / 3$ roll-off from $0.005 \mathrm{~s}^{-1}$ to $10 \mathrm{~s}^{-1}$, which approximates the Kolmogorov theory [25] for the inertial subrange of the power spectrum of the high Reynolds number turbulence that is typical for the atmosphere [17]. The second wind model is thus a coarse approximation for turbulence.

Example 1. Average power of pitch error and average power of altitude error as functions of $k$. Consider the flight scenario with a constant altitude command $h_{\mathrm{d}}(t) \equiv$ $h_{0}=100 \mathrm{~m}$ and where the wind disturbance is the Gaussian white-noise wind model. Figure 4 shows the average power $\mathcal{P}_{\theta}\left(t_{1}, t_{0}\right)$ of the pitch error and the average power $\mathcal{P}_{h}\left(t_{1}, t_{0}\right)$ of the altitude error for different values of $k$ and where $t_{0}=20 \mathrm{~s}$ and $t_{1}=100 \mathrm{~s}$. For FDI, $\mathcal{P}_{\theta}\left(t_{1}, t_{0}\right)$ decreases as $k$ increases. In fact, Fig. 4 suggests that $\mathcal{P}_{\theta}\left(t_{1}, t_{0}\right)$ can be made arbitrarily small for sufficiently large $k$, which supports the analytic results in Ref. [18, Theorem 1]. In addition, $\mathcal{P}_{h}\left(t_{1}, t_{0}\right)$ decreases as $k$ increases; however, Fig. 4 suggests that $\mathcal{P}_{h}\left(t_{1}, t_{0}\right)$ cannot be made arbitrarily small. Figure 4 also demonstrates that, for all $k>15, \mathcal{P}_{\theta}\left(t_{1}, t_{0}\right)$ and $\mathcal{P}_{h}\left(t_{1}, t_{0}\right)$ with the FDI controller (19) is less than that with the PI controller (20). The PI performance cannot be improved by increasing $k_{\theta, \mathrm{p}}$, because the closed-loop system is unstable if $k_{\theta, \mathrm{p}}>2.2$.

Example 2. Power spectral density with Gaussian white-noise wind. Consider the flight scenario with a constant altitude command $h_{\mathrm{d}}(t) \equiv h_{0}=100 \mathrm{~m}$ and where the wind disturbance is the Gaussian white-noise wind model. The time-domain pitch error and altitude error are each divided into 59 segments of $164 \mathrm{~s}$ with $82 \mathrm{~s}$ of overlap between time-adjacent segments. The frequency-domain
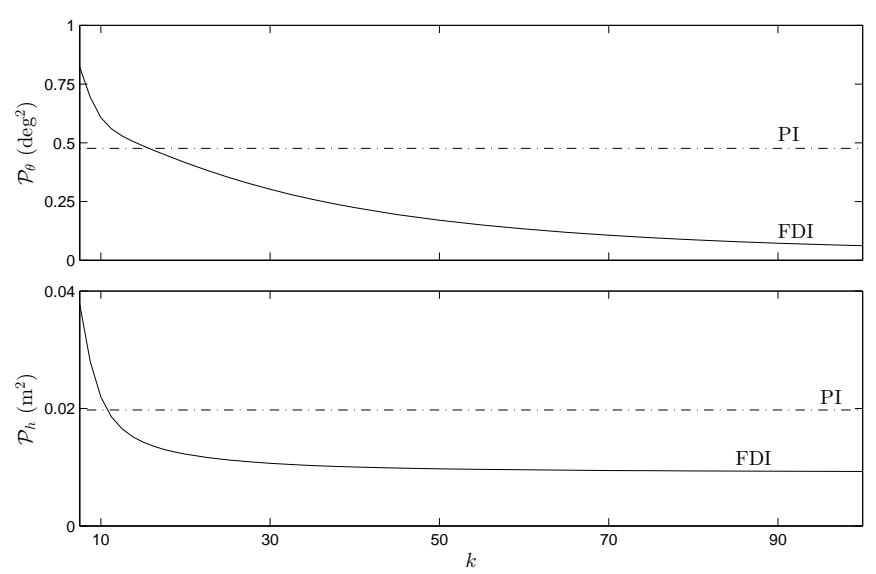

Figure 4: Average power of pitch error and average power of altitude error as functions of $k$. As $k$ increases, $\mathcal{P}_{\theta}\left(t_{1}, t_{0}\right)$ decreases and $\mathcal{P}_{h}\left(t_{1}, t_{0}\right)$ decreases. For $k>15$, the average power $\mathcal{P}_{\theta}\left(t_{1}, t_{0}\right)$ of the pitch error with the FDI controller is less than that with the PI controller. For $k>12$, the average power $\mathcal{P}_{h}\left(t_{1}, t_{0}\right)$ of the altitude error with the FDI controller is less than that with the PI controller.
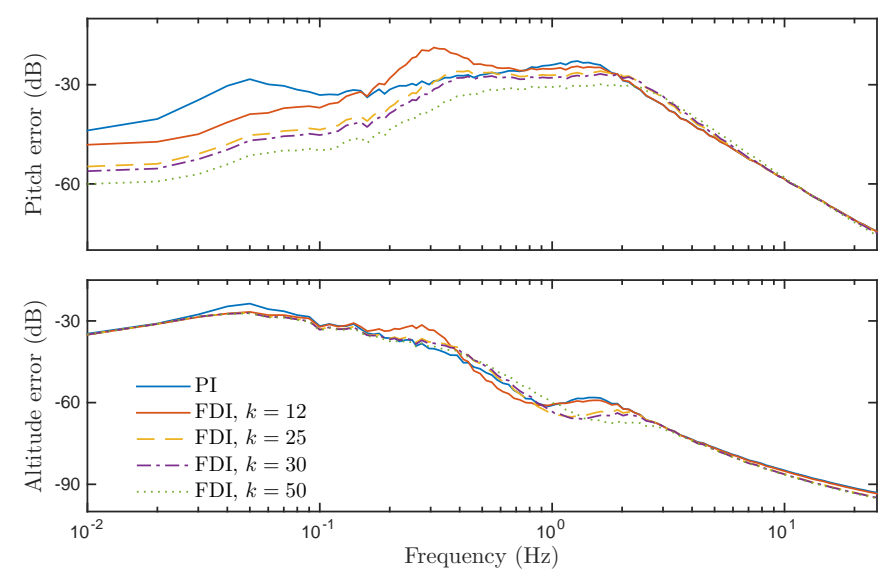

Figure 5: Pitch-error and altitude-error power spectral densities. The pitch-error power spectral density with the FDI controller (for $k \geq 25$ ) is lower than that with the PI controller for frequencies less than $2 \mathrm{~Hz}$. The altitude-error power spectral density with the FDI controller (for $k \geq 25$ ) is lower than that with the PI controller across most of the 0 -to- $25 \mathrm{~Hz}$ frequency range.

data are calculated by averaging the ratios of the discrete Fourier transforms from the 59 segments. Figure 5 shows the power spectral density of the pitch error and the altitude error. Increasing the FDI parameter $k$ tends to decrease the magnitude of the pitch-error power spectral density. For $k \geq 25$, the pitch-error power spectral density with the FDI controller is lower than that with the PI controller (20) at all frequencies less than $2 \mathrm{~Hz}$. Increasing the FDI parameter $k$ also tends to decrease the magnitude of the altitude-error power spectral density at certain frequencies. Specifically, for $k \geq 25$, the altitude-error power spectral density with FDI is lower than that with PI across the 1-to-2.5 $\mathrm{Hz}$ frequency range.

Example 3. Step altitude commands and turbulent wind. Consider the flight scenario where $h_{\mathrm{d}}$ is series of steps, filtered through the first-order, unity-DC-gain, lowpass filter $2 /(s+2)$ and where the wind disturbance is the turbulent wind model. The FDI parameter is $k=25$. Fig- 
ure 6 provides time histories of pitch $\theta$, pitch command $\theta_{\mathrm{d}}$, pitch error $\theta_{\mathrm{d}}-\theta$, altitude $h$, altitude command $h_{\mathrm{d}}$, altitude error $h_{\mathrm{d}}-h$, speed $U_{\mathrm{r}}$, and elevator command $u_{\mathrm{e}}$. For the FDI controller, $\mathcal{P}_{\theta}\left(t_{1}, t_{0}\right)=0.427 \mathrm{deg}^{2}$ and $\mathcal{P}_{h}\left(t_{1}, t_{0}\right)=5.69 \mathrm{~m}^{2}$, where $t_{0}=20 \mathrm{~s}$ and $t_{1}=100 \mathrm{~s}$. For the PI controller, $\mathcal{P}_{\theta}\left(t_{1}, t_{0}\right)=1.642 \mathrm{deg}^{2}$ and $\mathcal{P}_{h}\left(t_{1}, t_{0}\right)=$ $7.56 \mathrm{~m}^{2}$. The ratio of the PI average power of pitch error to the FDI average power of pitch error is 3.84 . The ratio of the PI average power of altitude error to the FDI average power of altitude error is 1.33. The FDI controller (19) improves $\mathcal{P}_{\theta}\left(t_{1}, t_{0}\right)$ and $\mathcal{P}_{h}\left(t_{1}, t_{0}\right)$ relative to the PI controller $(20)$.
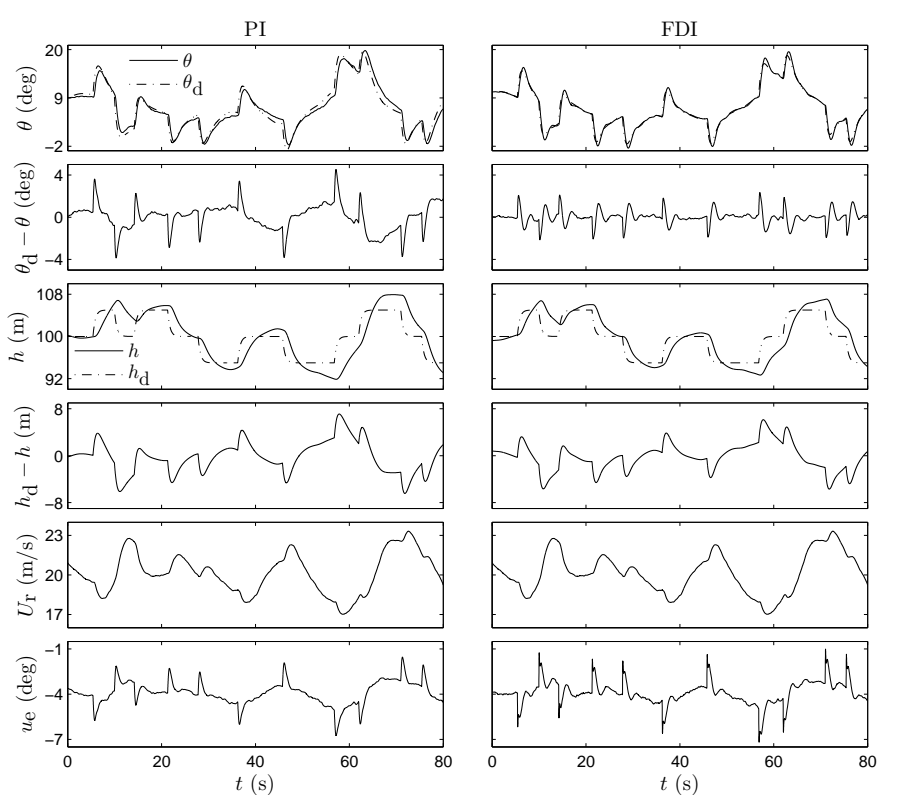

Figure 6: Step altitude commands and turbulent wind. The FDI controller exhibits better pitch command following and altitude command following than the PI controller.

\section{Discretization of the FDI Control System}

We now examine the impact of discretizing the FDI control system, given by Eqs. (9), (10), and (19), for implementation on the digital autopilot, which is described in Section 8. The digital autopilot and its sensor package has limited bandwidth, and thus, we examine digital implementation of Eqs. (9), (10), and (19) with sample frequencies no faster than $100 \mathrm{~Hz}$. In fact, the autopilot sensors provide data at a sample time of $0.02 \mathrm{~s}$ (i.e., a sample frequency of $50 \mathrm{~Hz}$ ). Each controller (9), (10), and (19) is discretized using a zero-order hold on the input and a uniform sample time $T_{\mathrm{s}}$. We now repeat Example 1 with the discrete-time FDI control. We use the nonlinear aircraft dynamics (7) and (8), and the same physical UAV parameters in Section 6.1.

Example 4. Average power of pitch error and average power of altitude error as functions of $k$ with discretetime FDI and sample time $T_{\mathrm{s}} \in\{0.01,0.02,0.04\} \mathrm{s}$. Consider the flight scenario with a constant altitude command $h_{\mathrm{d}}(t) \equiv h_{0}=100 \mathrm{~m}$ and where the wind disturbance is the Gaussian white-noise wind model. Figure 7 shows the average power $\mathcal{P}_{\theta}\left(t_{1}, t_{0}\right)$ of the pitch error and the average power $\mathcal{P}_{h}\left(t_{1}, t_{0}\right)$ of the altitude error as a function of $k$ for the discrete-time FDI controller with sample time $T_{\mathrm{s}} \in\{0.01,0.02,0.04\} \mathrm{s}$. Note that $t_{0}=20 \mathrm{~s}$ and $t_{1}=100 \mathrm{~s}$. Figure 7 demonstrates that for each $T_{\mathrm{s}}$, as $k$ increases, $\mathcal{P}_{\theta}\left(t_{1}, t_{0}\right)$ decreases until reaching a minimum. Increasing $k$ further, causes $\mathcal{P}_{\theta}\left(t_{1}, t_{0}\right)$ to increase and eventually leads to an unbounded pitch response (i.e., instability). However, for $T_{\mathrm{s}} \in\{0.01,0.02\} \mathrm{s}$, the discretetime FDI controller improves the average power $\mathcal{P}_{\theta}\left(t_{1}, t_{0}\right)$ of the pitch error relative to the PI controller. Note that reducing sample time $T_{\mathrm{s}}$ from $0.04 \mathrm{~s}$ to $0.01 \mathrm{~s}$, reduces the minimum value of $\mathcal{P}_{\theta}\left(t_{1}, t_{0}\right)$ and increases the associated value of $k$. Similarly, for $T_{\mathrm{s}} \in\{0.01,0.02,0.04\} \mathrm{s}$, the discrete-time FDI controller improves the average power $\mathcal{P}_{h}\left(t_{1}, t_{0}\right)$ of the altitude error relative to the PI controller. Furthermore, as $T_{\mathrm{s}}$ decreases, the $\mathcal{P}_{\theta}\left(t_{1}, t_{0}\right)$ versus $k$ plot and the $\mathcal{P}_{h}\left(t_{1}, t_{0}\right)$ versus $k$ plot for the discrete-time FDI controller approaches that of the continuous-time FDI controller.
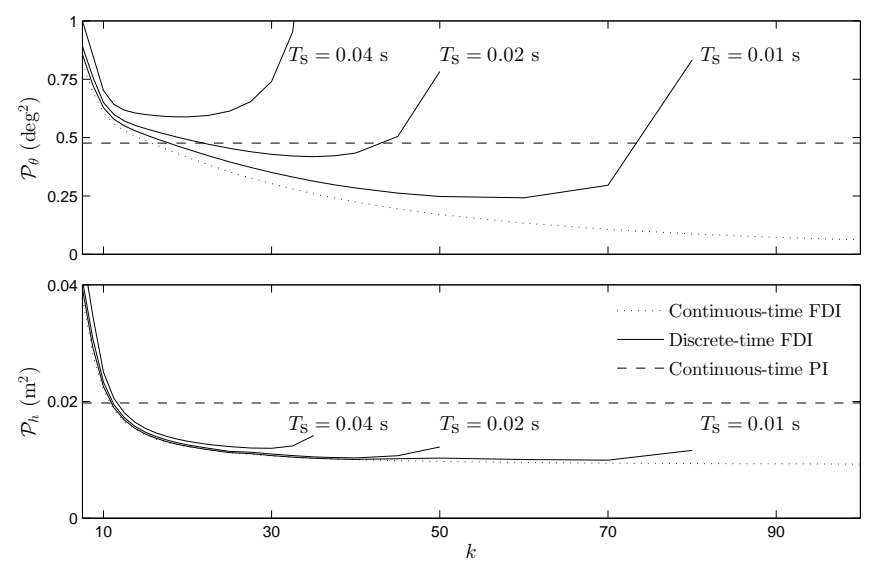

Figure 7: Average power of pitch error and average power of altitude error as functions of $k$ with discrete-time FDI and sample time $T_{\mathrm{s}} \in$ $\{0.01,0.02,0.04\} s$. For each $T_{\mathrm{s}}$, as $k$ increases, $\mathcal{P}_{\theta}\left(t_{1}, t_{0}\right)$ decreases, reaches a minimum, and then increases. For $T_{\mathrm{s}} \in\{0.01,0.02\} \mathrm{s}$, the discrete-time FDI controller can improve $\mathcal{P}_{\theta}\left(t_{1}, t_{0}\right)$ relative to the PI controller. For $T_{\mathrm{s}} \in\{0.01,0.02,0.04\} \mathrm{s}$, the discrete-time FDI controller can improve $\mathcal{P}_{h}\left(t_{1}, t_{0}\right)$ relative to the PI controller.

Example 4 demonstrates that for $T_{\mathrm{S}} \leq 0.02 \mathrm{~s}$, the discrete-time FDI controller can improve pitch and altitude command following relative to the PI controller. Repeating Example 3 with the continuous-time FDI controller (19) replaced by the discrete-time FDI controller with $T_{\mathrm{s}}=0.02 \mathrm{~s}$ results in closed-loop responses that are indistinguishable from the plots shown in Fig. 6.

\section{Altitude Control Experiment}

This section describes an experimental implementation of the FDI controller (19) and the PI controller (20) on the small fixed-wing UAV described in Section 6.1.

\subsection{UAV Autopilot Hardware}

The flight controller was an Ardupilot Mega 2.5, which is an open-source autopilot based on the Arduino computing platform. The Ardupilot featured an Invensense 
MPU-6000 six-axis accelerometer and gryoscope, Measurement Specialties MS5611-01BA03 barometer, Honeywell HMC5883L-TR magnetometer, and uBlox LEA-6H GPS system. A Pitot-static probe and pressure transducer provide airspeed sensing to measure $U_{\mathrm{r}}$. The pressure transducer was a Freescale Semiconductor MPXV7002, which had a $\pm 2 \mathrm{kPa}$ range, which approximately corresponds to a 0 -to- $55 \mathrm{~m} / \mathrm{s}$ sensing range for $U_{\mathrm{r}}$.

The Ardupilot operates using the Arduplane software package, which is open-source. The altitude $h$, latitude, longitude, speed $U_{\mathrm{r}}$, Euler angles $\phi, \theta, \psi$, and angular rates $P, Q, R$ are available for feedback. To implement the PI and FDI pitch controllers, we altered the pitch controller module of the Arduplane v2.74b source code. The revised function responsible for inputting a pitch error and outputting an elevator servomechanism command was implemented at $50 \mathrm{~Hz}$. The software was designed to allow switching between the PI and FDI pitch controllers in flight and to reset all states of the controllers to zero following the change of controller. Both the PI and FDI pitch controllers were discretized using a zero-order hold at $50 \mathrm{~Hz}$ and augmented with a discrete-time approach to prevent integrator windup from saturation of the elevator servomechanism. See Ref. [26] for the anti-windup approach.

To gather data, the Arduplane firmware was altered to $\log$ altitude $h$, altitude error $h_{\mathrm{d}}-h$, commanded elevator deflection $u_{\mathrm{e}}$, pitch command $\theta_{\mathrm{d}}$, and pitch $\theta$ at $50 \mathrm{~Hz}$. The UAV's distance from its next waypoint was also logged. During flight, the ground crew was able to monitor position, attitude, and speed; change the predefined tunable parameters using Mission Planner v1.3.1 software; and toggle between the FDI and PI pitch controllers.

\subsection{Flight Location and Flight Path}

Experiments were conducted at the Lexington Model Airplane Club Facilities located in Lexington, Kentucky. The field featured a paved runway that is approximately $200 \mathrm{~m}$ long, oriented WSW and ENE, to match the predominant spring-to-fall wind direction.

The objective of the test flights was to evaluate the altitude and pitch command-following performance of FDI compared to that of the PI controller. To minimize systematic errors in the flight data due to transient weather changes, each test flight consisted of one portion of the flight operated under PI control and one portion of the flight operated under FDI control. The flight controller that was used first during the test flight was randomly selected.

The clockwise flight path is also shown in Fig. 8, where the waypoints $\mathrm{A}$ through $\mathrm{H}$ were all $100 \mathrm{~m}$ above a constant reference ground elevation. A lap was defined as starting and ending at point A and took approximately one minute to complete. Each test flight consisted of six laps and began with the aircraft taking off in the WSW direction under manual control. Manual control was maintained as the aircraft gained altitude and turned to align approximately with the $\mathrm{H}-\mathrm{A}$ segment at approximately

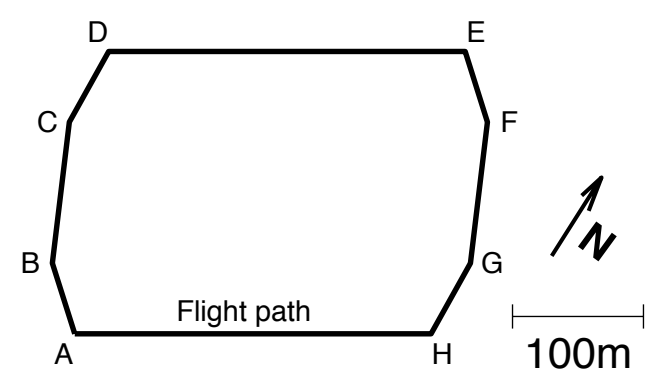

Figure 8: Flight Path. The experimental flightpath resembles a clockwise oval racetrack. The flight path is designed such that the aircraft is flying straight and level for as long as possible.

$100 \mathrm{~m}$ altitude. The aircraft was then switched into automatic control, using either the PI or FDI inner pitch control loop, before reaching waypoint A. The first lap was completed under autonomous flight control; however, this lap was excluded from the analysis to minimize the influence of initial conditions introduced by the manual control. The second and third laps (beginning and ending at waypoint A) were used for analysis of the first controller. Between waypoints D and $\mathrm{E}$ on the fourth lap, the pitch controller was changed from PI to FDI or FDI to PI. This fourth lap was also excluded from analysis. The fifth and sixth laps (beginning and ending at waypoint A) were performed under autonomous flight and were used for analysis of the second controller. Following completion of the sixth lap at waypoint A, control was returned to the pilot who landed the aircraft on the runway in the WSW direction.

During autonomous flight, telemetry data was monitored by the ground crew to ensure nominal operation of the UAV. However, these data were transmitted at intervals which were dependent on communication quality. Therefore, data analysis was performed using the data logged to on-board memory by the autopilot at the fixed $50 \mathrm{~Hz}$ rate. These data were downloaded to the ground station following completion of each test flight. In processing the data, the distance-to-waypoint data were used to extract individual measurement segments of the data corresponding to each controller.

All flight tests reported in this paper were conducted on Tuesday May 27, 2014 in clear weather. Flights 1, 2, and 3 had relatively constant winds from the WSW direction at 7 knots, whereas Flight 4 had relatively constant winds from the $\mathrm{W}$ direction at 12 knots. All flight tests used the same pitch and altitude controller parameters as in the simulations. Specifically, we used the FDI parameters $\eta_{k}(s)=(s+k)^{4}, \alpha_{\mathrm{m}}(s)=\beta_{\mathrm{m}}(s)=(s+4)(s+6)(s+8)$, $\bar{H}_{d}=1,005$, and $\operatorname{sgn}\left(H_{d}\right)=-1$; the PI pitch control parameters $k_{\theta, \mathrm{p}}=0.5$ and $k_{\theta, \mathrm{i}}=-0.1$; and the altitude control parameters $k_{\mathrm{h}, \mathrm{P}}=1.6$ and $k_{\mathrm{h}, \mathrm{I}}=0.2$. The FDI parameter $k$ was different for each flight.

\subsection{Experimental Results}

Flights 1, 2, 3, and 4 were conducted with the FDI parameters $k=12, k=25, k=30$, and $k=30$, respectively. Time histories of the pitch angle $\theta$, pitch error $\theta_{\mathrm{d}}-\theta$, altitude $h$, aircraft speed $U_{\mathrm{r}}$, and elevator servo input $u_{\mathrm{e}}$ from 

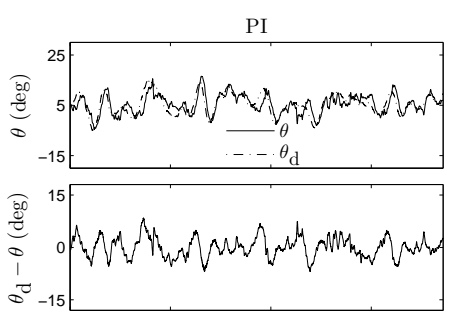

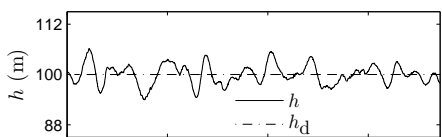
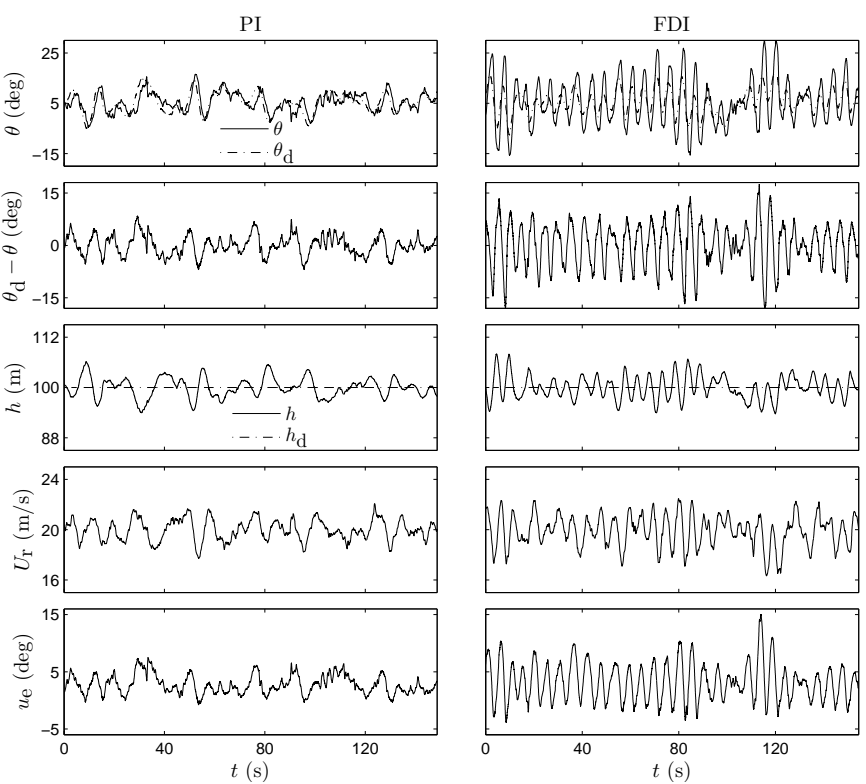

Figure 9: Time Histories for Flight $1(k=12)$. Neither the pitch error nor altitude error with FDI is reduced relative to that with PI.
PI

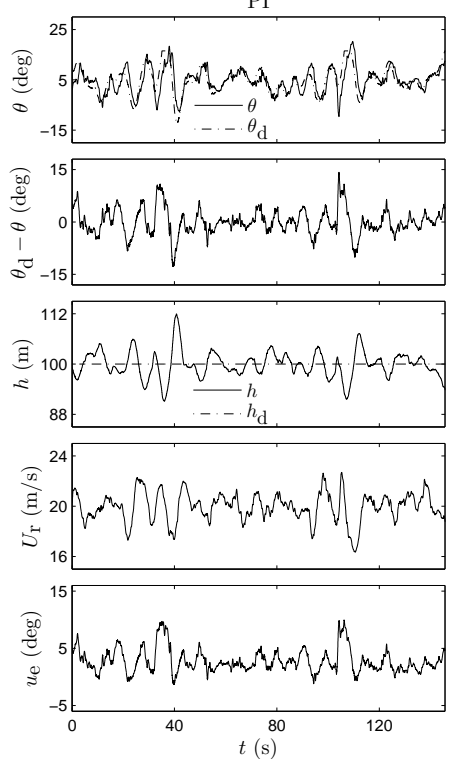

FD
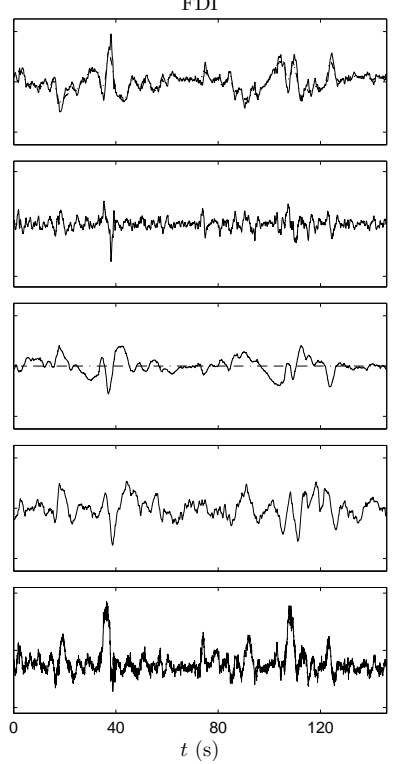

Figure 10: Time Histories for Flight $2(k=25)$. The pitch error and altitude error with FDI are reduced relative to that with PI.

the measurement portions of each test flight are shown in Figs. 9, 10, 11 and 12 for Flights 1, 2, 3, and 4, respectively. The corresponding discrete power spectral densities of the pitch error and altitude error are shown in Figs. 13, 14,15 and 16 . The average power $\mathcal{P}_{\theta}\left(t_{1}, t_{0}\right)$ of the pitch error and the average power $\mathcal{P}_{h}\left(t_{1}, t_{0}\right)$ of the altitude error is examined for all flights, where $t_{1}-t_{0}=150 \mathrm{~s}$. For brevity, the arguments $t_{1}$ and $t_{0}$ are omitted.

For $k=12$, the pitch and altitude response demonstrated low-frequency oscillations at approximately $0.2 \mathrm{~Hz}$ as shown in Fig. 9. As a result, the PI controller displayed significantly better pitch command following, with $\left|\theta_{\mathrm{d}}-\theta\right|<6^{\circ}$ for PI compared to $\left|\theta_{\mathrm{d}}-\theta\right|>12^{\circ}$ for FDI. Interestingly, the maximum altitude deviations for both FDI and PI were within the $\pm 7 \mathrm{~m}$ of the target altitude of 100
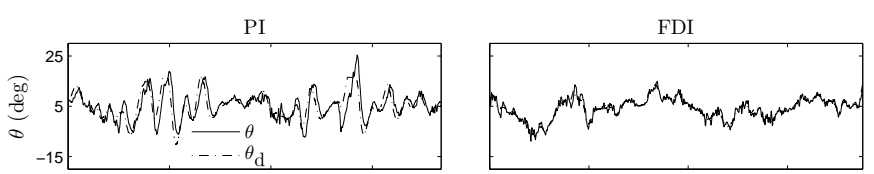

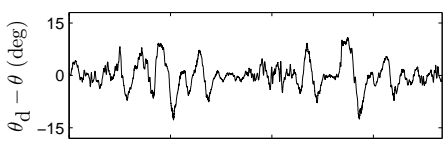

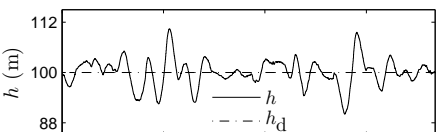
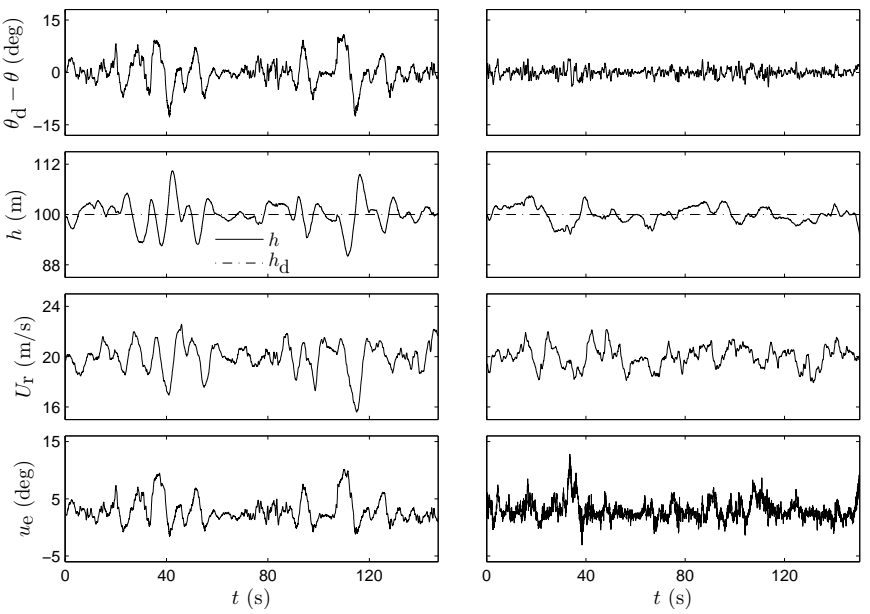

Figure 11: Time Histories for Flight $3(k=30)$. The pitch error and altitude error with FDI are reduced relative to that with PI.
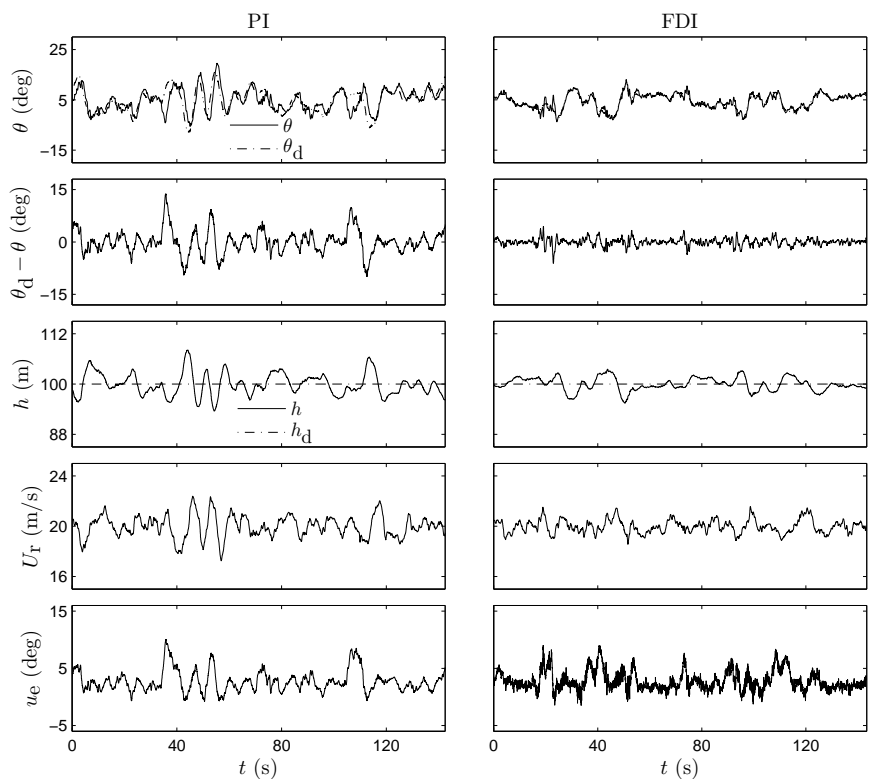

Figure 12: Time Histories for Flight $4(k=30)$. The pitch error and altitude error with FDI are reduced relative to that with PI.

$\mathrm{m}$; however, the FDI controller had more frequent excursions from the target range. As a result $\mathcal{P}_{\theta}=40.09 \mathrm{deg}^{2}$ for FDI, whereas $\mathcal{P}_{\theta}=8.05 \mathrm{deg}^{2}$ for PI. This resulted in $\mathcal{P}_{h}=7.97 \mathrm{~m}^{2}$ for FDI, and $\mathcal{P}_{h}=5.60 \mathrm{~m}^{2}$ for PI. Thus, at $k=12$, the ratio of the PI average power of pitch error to the FDI average power of pitch error was 0.20 , and the ratio for the average power of the altitude error was 0.70 . As shown in Fig. 13, the FDI controller's worse performance was largely due to the $0.2 \mathrm{~Hz}$ oscillations. In fact, even at the relatively low value $k=12$, the FDI controller demonstrated improved pitch command following at low frequency (i.e., less than $0.1 \mathrm{~Hz}$ ). This, however, did not result in improved altitude tracking at low frequency.

For $k=25$, the pitch and altitude command following of the FDI controller improved significantly. As shown in 

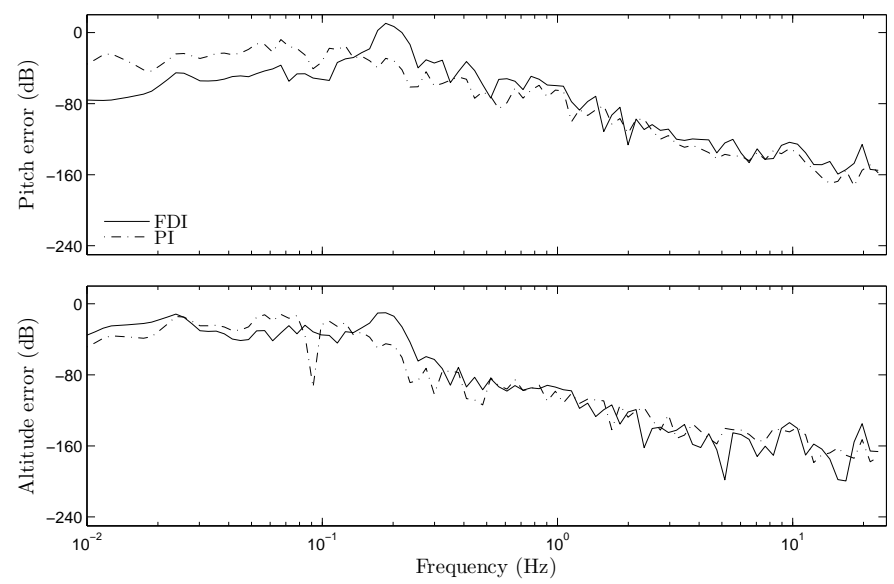

Figure 13: Power Spectral Densities for Flight $1(k=12)$. The magnitude of the pitch error with FDI is smaller than that with PI at low frequency (i.e., less than $0.1 \mathrm{~Hz}$ ). However, the magnitude of the pitch error with FDI is larger than that with PI at frequencies above $0.2 \mathrm{~Hz}$. For the altitude error, the magnitude with FDI is larger than that with PI at almost all frequencies.
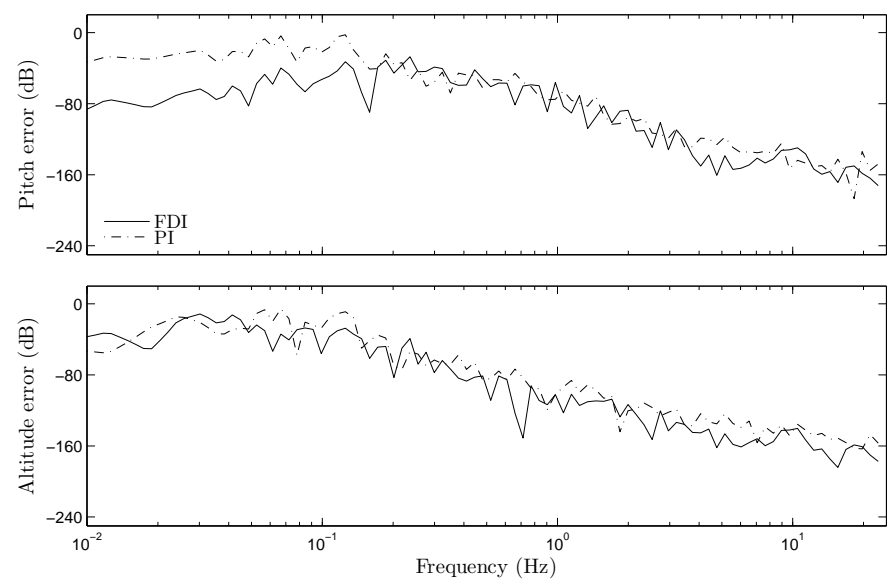

Figure 14: Power Spectral Densities for Flight $2(k=25)$. The magnitude of the pitch error with FDI is smaller than that with PI below $0.2 \mathrm{~Hz}$. For the altitude error, the magnitude with FDI is smaller than that with PI at almost all frequencies.

Fig. 10, the excursions of $\theta_{\mathrm{d}}-\theta$ are typically under $\pm 4^{\circ}$ for FDI compared to $\pm 7^{\circ}$ for PI. This resulted in lower amplitude excursions in altitude as well. However, as can be observed in the time series of $u_{e}$, to accomplish this there is more demand for actuation at higher frequencies. For FDI, $\mathcal{P}_{\theta}=2.62 \mathrm{deg}^{2}$, and for PI, $\mathcal{P}_{\theta}=13.71 \mathrm{deg}^{2}$, resulting in a ratio of 5.23 . Similarly, for FDI, $\mathcal{P}_{h}=3.93 \mathrm{~m}^{2}$, and for PI, $\mathcal{P}_{h}=10.14 \mathrm{~m}^{2}$, resulting in a ratio of 2.58 . As shown in Fig. 14, the improvement in pitch command following occurred primarily at frequencies less than $0.2 \mathrm{~Hz}$. Conversely, the improvement in altitude command following occurred at frequencies greater than $1 \mathrm{~Hz}$.

Two flight tests were completed at the highest value $k=30$. In both cases, the FDI controller demonstrated improvements over the PI controller with trends similar to those observed in the $k=25$ flight test as shown in Figs. 11 and 12. The higher value of $k$ resulted in further reduction of the average power of pitch error and the average power of altitude error. For FDI, $\mathcal{P}_{\theta}$ was reduced to $1.38 \mathrm{deg}^{2}$ for Flight 3 and $1.09 \mathrm{deg}^{2}$ for Flight 4 compared
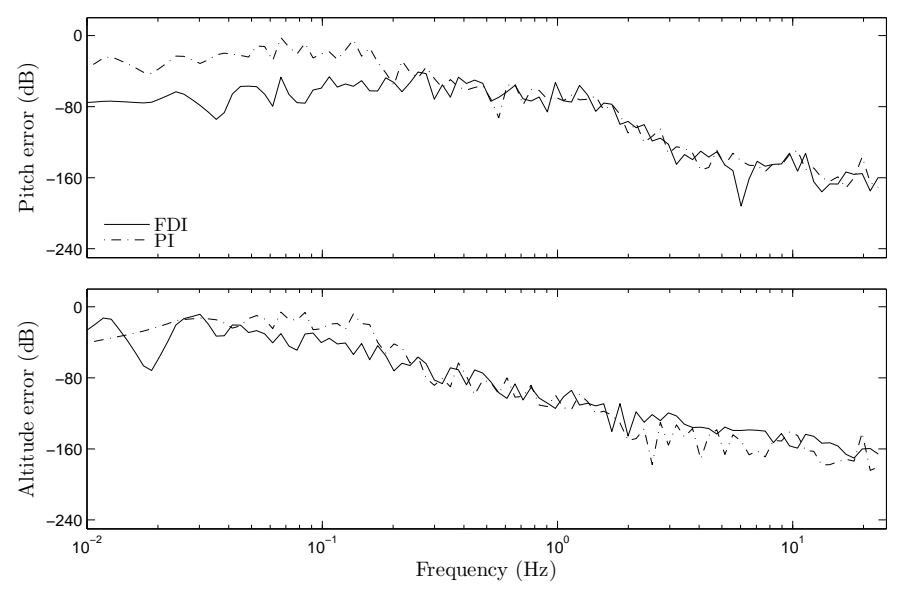

Figure 15: Power Spectral Densities for Flight $3(k=30)$. The magnitude of the pitch error with FDI is smaller than that with PI below $0.2 \mathrm{~Hz}$. For the altitude error, the magnitude with FDI is smaller than that with PI at almost all frequencies.
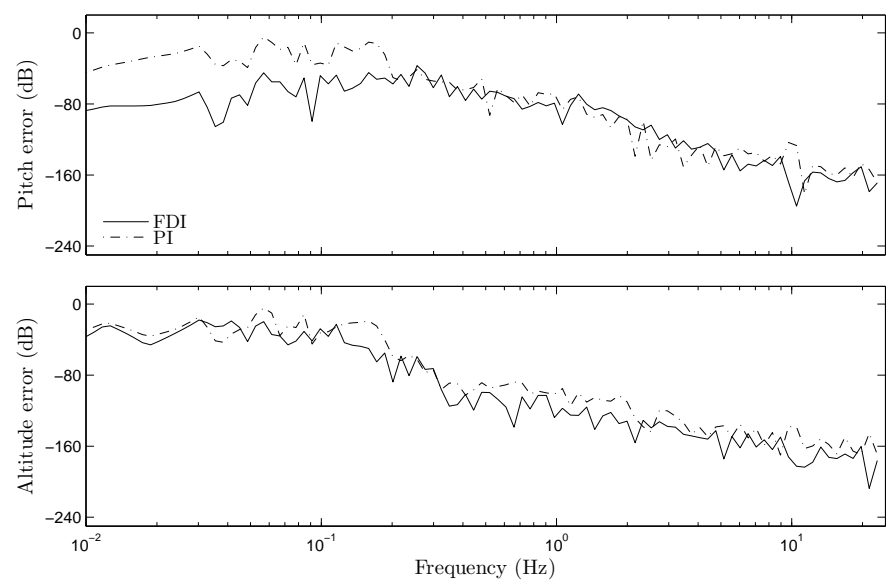

Figure 16: Power Spectral Densities for Flight $4(k=30)$. The magnitude of the pitch error with FDI is smaller than that with PI below $0.2 \mathrm{~Hz}$. For the altitude error, the magnitude with FDI is smaller than that with PI at almost all frequencies.

to the PI values of $14.47 \mathrm{deg}^{2}$ for Flight 3 and $11.16 \mathrm{deg}^{2}$ for Flight 4 . The ratio between the values of $\mathcal{P}_{\theta}$ was 10.52 and 10.21 for Flight 3 and Flight 4, respectively. Similar reductions were observed in $\mathcal{P}_{h}$. For FDI, $\mathcal{P}_{h}$ was $3.60 \mathrm{~m}^{2}$ and $2.47 \mathrm{~m}^{2}$ for Flight 3 and Flight 4 compared to the PI values of $10.90 \mathrm{~m}^{2}$ and $7.09 \mathrm{~m}^{2}$. The ratios between the values of $\mathcal{P}_{h}$ was 3.03 and 2.87 for Flight 3 and Flight 4 , respectively. The corresponding discrete power spectral densities of the measured pitch and altitude error shown in Figs. 15 and 16 demonstrate that the improved pitch command following occurred primarily at frequencies less than $0.2 \mathrm{~Hz}$, whereas the improved altitude command following occurred at low frequency for Flight 3 and across the entire frequency range for Flight 4.

These results are summarized in Table 1, which shows $\mathcal{P}_{\theta}$ and $\mathcal{P}_{h}$ for each flight. In analyzing the results, we assume that the atmospheric turbulence did not change appreciably during a single flight but may have changed between flights. This assumption is supported by the repeated test case of $k=30$, which produced only $3.0 \%$ difference between measured $\mathcal{P}_{\theta}$ ratios and $5.4 \%$ difference 
between measured $\mathcal{P}_{h}$ ratios, despite an approximately $20 \%$ difference in $\mathcal{P}_{\theta}$ and $\mathcal{P}_{h}$ between the two flights.

\begin{tabular}{c|c|c|c|c|c|c|c}
\multicolumn{2}{l|}{} & \multicolumn{3}{c|}{$\mathcal{P}_{\theta}\left(\mathrm{deg}^{2}\right)$} & \multicolumn{3}{c}{$\mathcal{P}_{h}\left(\mathrm{~m}^{2}\right)$} \\
\hline Flight & $k$ & PI & FDI & Ratio & PI & FDI & Ratio \\
\hline 1 & 12 & 8.05 & 40.09 & 0.20 & 5.60 & 7.97 & 0.70 \\
2 & 25 & 13.71 & 2.62 & 5.23 & 10.14 & 3.93 & 2.58 \\
3 & 30 & 14.47 & 1.38 & 10.52 & 10.90 & 3.60 & 3.03 \\
4 & 30 & 11.16 & 1.09 & 10.21 & 7.09 & 2.47 & 2.87
\end{tabular}

Table 1: Summary of Experimental Flight Results.

The frequency-domain results are consistent with the numerically predicted response shown in Fig. 5. Notably, for $k=12$, the experimental and numerical pitch errors both shows an increase in frequency content for FDI relative to PI from 0.1 to $0.3 \mathrm{~Hz}$, and a significant decrease in frequency content for FDI relative to PI at low frequencies (i.e., less than $0.1 \mathrm{~Hz}$ ). In addition, the experimental lowfrequency (i.e., less than $0.2 \mathrm{~Hz}$ ) pitch error is smaller for larger values of $k$. This is consistent with the numerical results in Fig. 5.

Comparing the experimental PI-to-FDI ratios for $\mathcal{P}_{\theta}$ and $\mathcal{P}_{h}$, we note that the ratios are larger for larger $k$, in accordance with the predictions of the numerical simulations in Section 7. Qualitatively, the $k$ dependence of the experimental ratios is consistent with the numerical predictions. However, quantitatively, the experiment shows larger PIto-FDI ratios than the numerical simulations, indicating that the FDI improvement over PI is more pronounced in the experimental results. One possible source of variation between the experimental and numerical results is airspeed error, which is coupled to the UAV pitch dynamics and a simplified PI speed controller is implemented in the numerical simulations. In the experiment, the average power of the airspeed error for PI, ranged from 0.76 to $1.63 \mathrm{~m}^{2} / \mathrm{s}^{2}$, whereas for FDI, it ranged from 0.28 to $0.72 \mathrm{~m}^{2} / \mathrm{s}^{2}$. Hence, the decreased performance of the PI pitch controller could have been accentuated by the throttle control.

The flight path is another difference between simulation and experiment. The simulations used roll control but did not implement a heading controller. In the simulations, the roll angle was regulated to zero (i.e., level flight). In the experiment, a guidance loop provided roll commands based on heading error. Thus, if FDI performance is superior to PI for non-level flight, then the nonzero experimental UAV roll could help explain the improved experimental performance of FDI relative to PI.

Differences between the estimated value of $\bar{H}_{d}$ and the actual experimental value could cause changes in the average powers of performance for FDI. Underestimating $\bar{H}_{d}$ can change the high- $k$-stabilizing nature of FDI, while overestimating $\bar{H}_{d}$ tends to reduce the value of $k$ needed to achieve a prescribed level of performance.

\section{Conclusions}

Small fixed-wing UAVs have significant potential for scientific meteorological investigations. Maximizing the utility of data acquired by onboard sensors will require improvements in gust-rejection capability of the aircraft. One avenue for improving gust rejection is through improvements in the flight controller. In this paper, FDI is implemented in the altitude control loop of the flight controller of a small fixed-wing UAV. The FDI performance is compared to that of a classical PI controller. One key advantage of FDI for this application is that it requires limited model information and is effective for command following in the presence of unmeasured disturbances.

Simulations using a nonlinear UAV model demonstrated that, for values of the FDI parameter $k>15$, there was a noticeable decrease in the average power of altitude error compared to that of the PI controller, with a reduction in average power of altitude error of approximately $50 \%$ achieved for $k>30$. These improvements were observed in the form of improved rejection of disturbances at high frequencies. Similar performance was observed when simulations where conducted using a discretized FDI controller, except that the maximum value of $k$ which can be stably implemented is limited by the sample time.

Experiments were also conducted using a small fixedwing UAV consisting of a low-cost, open-source autopilot integrated into a commercial off-the-shelf remote-control airframe. Test flights were conducted using both the PI and FDI control, with different values of $k$ tested. Measurements of average power of altitude error demonstrated the same trends as observed in the simulations, namely, a decrease in error with increasing $k$. Comparison between PI and FDI power spectral densities of altitude error were also qualitatively similar to those observed in simulations. Quantitatively, the difference between simulation and experiment came in the form of an improvement in the observed performance of the FDI controller relative to the PI controller in experiment, with a $65 \%$ reduction in average power of altitude error observed when $k=30$, coming predominantly at high frequency. Differences between the simulation and experiment are believed to be due to a combination of differences in the implementation of the throttle controller, differences in flight path, and differences between the estimated and actual value of the first nonzero Markov parameter.

These results demonstrate that measurable performance improvement is possible for small fixed-wing UAVs, and this improvement can enhance the feasibility of small UAVs as a low-cost meteorological test platform. In addition, the results demonstrate the suitability of FDI for making these performance improvements.

Acknowledgments. This work was supported by the National Science Foundation through CBET-1351411 and CMMI-1539070.

\section{References}

[1] C. Eheim, C. Dixon, B. M. Agrow, and S. Palo. Tornado chaser: A remotely-piloted UAV for in situ meteorological measurements. In Proceedings of 1 st AIAA Unmanned Aerospace Vehicles, Systems, Technologies, and Operations Conference and Workshop. Paper 2002-3479, 2002.

[2] S. Mayer, M. Jonassen, A. Sandvik, and J. Reuder. Atmospheric profiling with the UAS SUMO: a new perspective for 
the evaluation of fine-scale atmospheric models. Meteorology and Atmospheric Physics, 116(1-2):15-26, 2012.

[3] T. Bonin, P. Chilson, B. Zielke, and E. Federovich. Observations of the early evening boundary-layer transition using a small unmanned aerial system. Boundary-Layer Meteorol., 146:119-132, 2013.

[4] C. E. Wainwright, T. A. Bonin, P. B. Chilson, J. A. Gibbs, E. Fedorovich, and R. D. Palmer. Methods for evaluating the temperature structure-function parameter using unmanned aerial systems and large-eddy simulation. Boundary-Layer Meteorology, 155(2):189-208, 2015.

[5] T. A. Bonin, D. C. Goines, A. K. Scott, C. E. Wainwright, J. A. Gibbs, and P. B. Chilson. Measurements of the temperature structure-function parameters with a small unmanned aerial system compared with a sodar. Boundary-Layer Meteorology, 155(3):417-434, 2015.

[6] D. H. Lenschow and W. B. Johnson. Concurrent airplane and balloon measurments of atmospheric boundary layer structure over a forest. J. Appl. Meteor., 7:79-89, 1968.

[7] W. M. Angevine, S. K. Avery, and J. L. Kok. Virtual heat flux measurements from a boundary-layer profiler-rass compared to aircraft measurements. J. Appl. Meteor., 32:1901-1907, 1993.

[8] C. R. Philbrick. Raman lidar descriptions of lower atmosphere processes. In Proceedings of the 21st ILRC, pages 535-545, Valcartier, Quebec Canada, 2002.

[9] W. L. Eberhard, R. E. Cupp, and K. R. Healey. Doppler lidar measurement of profiles of turbulence and momentum flux. $J$. Atmos. Oceanic Technol., 6:809-819, 1989.

[10] V. Matvev, U. Dayan, I. Tass, and M. Peleg. Atmospheric sulfur flux rates to and from israel. The Science of the Total Environment, 291:143-154, 2002.

[11] S. Metzger, W. Junkermann, K. Butterbach-Bahl, H. P. Schmid, and T. Foken. Measuring the 3 -d wind vector with a weightshiftmicrolight aircraft. Atmos. Meas. Tech., 4:1421-1444, 2011.

[12] J. Egger, S. Bajrachaya, R. Heingrich, P. Kolb, S. Lammlein, M. Mech, J. Reuder, W. Schäper, P. Shakya, J. Shween, and H. Wendt. Diurnal winds in the himalayan kali gandaki valley. part iii: Remotely piloted aircraft soundings. Mon. Wea. Rev., 130:2042-2058, 2002.

[13] S. Hobbs, D. Dyer, D. Courault, A. Olioso, J.-P. Lagouarde, Y. Kerr, J. McAnneney, and J. Bonnefond. Surface layer profiles of air temperature and humidity measured from unmanned aircraft. Agronomie, 22(6):635-640, 2002.

[14] A. C. van den Kroonenberg, T. Spieß, M. Buschmann, T. Martin, P. S. Anderson, F. Beyrich, and J. Bange. Boundary layer measurements with the autonomous mini-UAV $\mathrm{M}^{2} \mathrm{AV}$. In Proceedings of DACH2007, Hamburg, Germany, 2007.

[15] A. C. van den Kroonenberg, T. Martin, M. Buschmann, J. Bange, and P. Vörsmann. Measuring the wind vector using the autonomous mini aerial vehicle $\mathrm{M}^{2} \mathrm{AV}$. J. Atmos. Oceanic Technol., 25:1969-1982, 2008.

[16] A. C. van den Kroonenberg, S. Martin, F. Beyrich, and J. Bange. Spatially-averaged temperature structure parameter over a heterogeneous surface measured by an unmanned aerial vehicle. Boundary-Layer Meteorology, 142(1):55-77, 2012.

[17] R. B. Stull. An Introduction to Boundary Layer Meteorology. Springer, 1988.

[18] J. B. Hoagg and T. M. Seigler. Filtered-dynamic-inversion control for unknown minimum-phase systems with unknown-andunmeasured disturbances. International Journal of Control, 86(3):449-468, 2013.

[19] T. M. Seigler and J. B. Hoagg. Filtered dynamic inversion for vibration control of structures with uncertainty. Journal of Dynamic Systems, Measurement, and Control, 135(4):041017.116, 2013.

[20] J. B. Hoagg and T. M. Seigler. Filtered feedback linearization for nonlinear systems with unknown disturbances. Sys. Contr. Lett., 62(8):613-625, 2013.

[21] B. Etkin. Dynamics of Atmospheric Flight. Dover Publications, 2000.

[22] W. Gracey. The experimental determination of the moments of inertia of airplanes by a simplified compound-pendulum method. Technical Report 1629, Washington, DC, 1948.

[23] J. Mullen. Filtered-dynamic-inversion control for fixed-wing unmanned aerial systems. Master's thesis, University of Kentucky,
2014.

[24] J. H. Blakelock. Automatic Control of Aircraft and Missiles. Wiley, 1965.

[25] A. N. Kolmogorov. The local structure of turbulence in incompressible viscous fluid for very large reynolds numbers. Dokl. Akad. Nauk SSSR, 30:301-305, 1941.

[26] S. Hodel and C. E. Hall. Variable-structure PID control to prevent integrator windup. IEEE Transactions on Industrial Electronics, 48(2):442-451, 2001. 